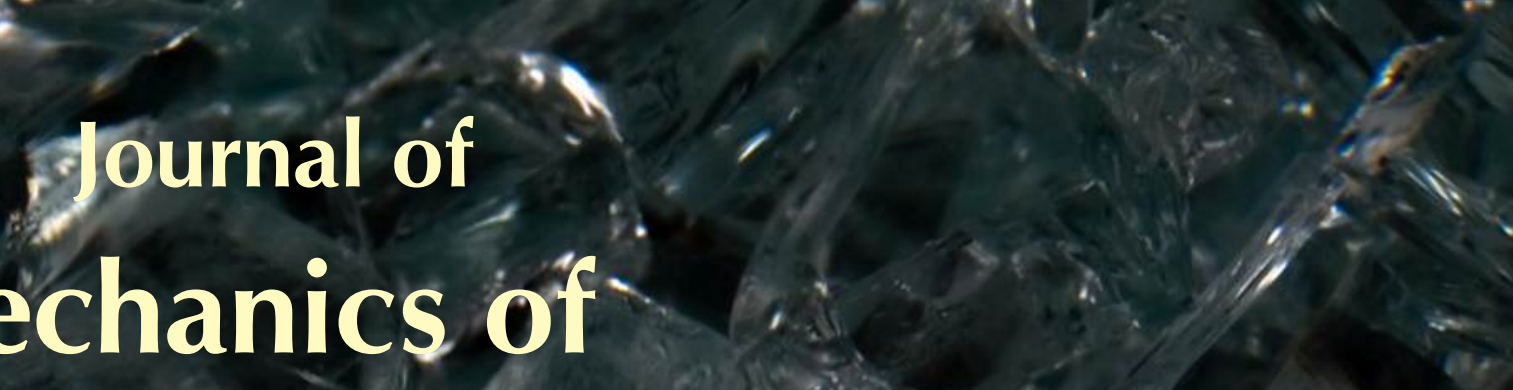

Materials and Structures

Mechanics of

P. BY A GALERKIN METHOD, WITHUUTT INVERTING ITHE TANGENT STIFFNESS MATRIX

Honghua Dai, Xiaokui Yue and Satya N. Atluri

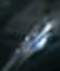

Volumeg, No. 2
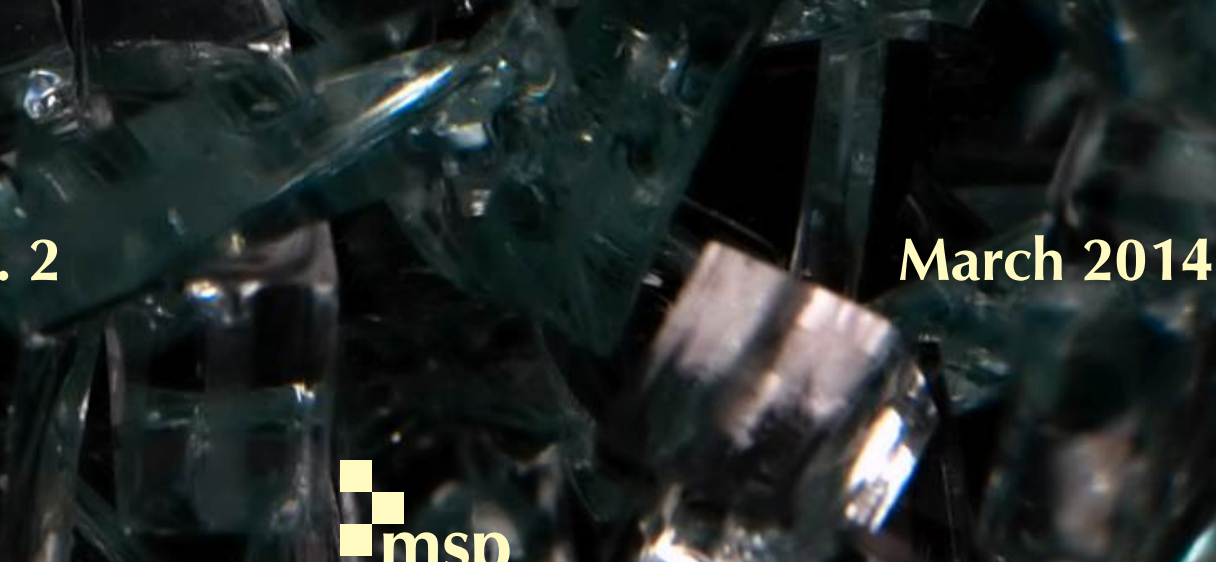


\title{
SOLUTIONS OF THE VON KÁRMÁN PLATE EQUATIONS BY A GALERKIN METHOD, WITHOUT INVERTING THE TANGENT STIFFNESS MATRIX
}

\author{
Honghua Dai, Xiaokui Yue and Satya N. Atluri
}

\begin{abstract}
Large deflections of a simply supported von Kármán plate with imperfect initial deflections, under a combination of in-plane loads and lateral pressure, are analyzed by a semianalytical global Galerkin method. While many may argue that the dominance of the finite element method in the marketplace may make any other attempts to solve nonlinear plate problems to be redundant and obsolete, semi- and precise analytical methods, when possible, simply serve as benchmark solutions if nothing else. Also, since parametric variations are simpler to access through such analytical methods, they are more useful in studying the physics of the phenomena. In the present method, the Galerkin scheme is first applied to transform the governing nonlinear partial differential equations of the von Kármán plate into a system of general nonlinear algebraic equations (NAEs) in an explicit form. The Jacobian matrix, the tangent stiffness matrix of the system of NAEs, is explicitly derived, which speeds up the Newton-Raphson iterative method if it is used. The present global Galerkin method is compared with the incremental Galerkin method, the perturbation method, the finite element method and the finite difference method in solving the von Kármán plate equations to compare their relative accuracies and efficiencies. Buckling behavior and jump phenomenon of the plate are detected and analyzed. Besides the classical NewtonRaphson method, an entirely novel series of scalar homotopy methods, which do not need to invert the Jacobian matrix (the tangent stiffness matrix), even in an elastostatic problem, and which are insensitive to the guesses of the initial solution, are introduced. Furthermore, we provide a comprehensive review of the newly developed scalar homotopy methods, and incorporate them into a uniform framework, which renders a clear and concise understanding of the scalar homotopy methods. In addition, the performance of various scalar homotopy methods is evaluated through solving the Galerkin-resulting NAEs. The present scalar homotopy methods are advantageous when the system of NAEs is very large in size, when the inversion of the Jacobian may be avoided altogether, when the Jacobian is nearly singular, and the sensitivity to the initially guessed solution as in the Newton-Raphson method needs to be avoided, and when the system of NAEs is either over- or under-determined.
\end{abstract}

\section{Introduction}

Analysis of large deflections of square and rectangular plates is one of the most studied engineering problems in the structural community, with many engineering applications including in aircraft structures, shipbuilding, bridges, and spaceships. The thin plates used in aircraft construction are subjected to lateral loads from the pressurized cabin or from the lift on the wings, and to edge loading due to bending of the fuselage and wings. The skin plates of a ship bottom are subjected to a significant water pressure, and to

Keywords: von Kármán plate equations, initial imperfection, global Galerkin method, nonlinear algebraic equations, scalar homotopy methods, buckling behavior. 
the edge loading owing to bending of the hull. Plate bending problems are also applicable to spaceships, where the outer plates may undergo lateral pressures and in-plane loadings.

The classical Kirchhoff theory for linear plate bending is accurate only for small deflection problems ( $w \leq 0.2$ thickness) ignoring the middle surface strains and the corresponding in-plane stresses. As the external force increases, the lateral deflection may be relatively large ( $w \geq 0.3$ thickness). In this scenario, the stretching of the middle surface of the plate should be considered and correspondingly the membrane forces arising from this stretching play a role in carrying lateral loads. The extension to large deformations was first provided by von Kármán in a seminal work [1910], wherein the nonlinear terms are retained in the kinematic relationships to account for a significantly large deflection of the plate $(w$ is comparable with plate thickness or larger but remains small with respect to other dimensions of the plate). This leads to a pair of coupled nonlinear fourth-order equations for the transverse displacement, and the stress function for the in-plane stress resultants. Inasmuch as the nonlinear terms are included in the coupled PDEs, closed form solutions for the nonlinear problem do not exist.

The first attempt to solve the von Kármán plate equations by a semianalytical method is attributed to Way [1939], who analyzed a geometrically nonlinear clamped rectangular plate via an energy method to obtain approximate solutions. Levy [1942a; 1942b] applied a Fourier series method to solve a simply supported rectangular plate under combined edge compression and lateral loading. Then Levy [1944] and Woolley et al. [1946] analyzed long rectangular plates with simply supported edges and clamped edges respectively by a similar approach. Okada, Oshima and Fukumoto [Okada et al. 1979] applied the Rayleigh-Ritz method to a simply supported long rectangular plate (length/width $=3$ and 4 ) under hydrostatic pressure, and discussed various buckling behaviors. Ueda, Rashed and Paik [Ueda et al. 1987] proposed an incremental Galerkin method by solving stepwise the linearized form of the von Kármán plate equations of the simply supported rectangular plate. The incremental Galerkin method was then applied to solve stiffened ship plates [Paik et al. 2001]. Large deflection of a simply supported plate was also analyzed in [Shen 1989] using a perturbation method and in [Bert et al. 1989] using a differential quadrature method. These semianalytical methods have respective drawbacks in that they may be too complex mathematically, or require large amounts of computational efforts, or have a slow convergence rate of the solution. Readers are advised to see [Chia 1980] for a comprehensive review.

With the development of modern digital computers, numerical methods based on domain discretization took over the difficult task. Brown and Harvey [1969] used the finite difference method to carry out the analysis of large deflections of rectangular plates subjected to a combination of lateral pressure and edge loading. Some of the earlier finite element implementations for large deformations were conducted in [Brebbia and Connor 1969] and [Bergan and Clough 1973], and involved conventional displacement based elements with the strain energy expressed in terms of the three displacement components. The stress based finite element method was proposed in [Punch and Atluri 1986]. A boundary element approach was proposed to investigate static, dynamic and buckling behavior of thin flat plates in [O'Donoghue and Atluri 1987]. Although these numerical methods can be employed to solve the von Kármán plate equations accurately and are more flexible than the semianalytical methods with respect to various boundary conditions and geometries, these domain discretization methods would require several orders of magnitude more degrees of freedom than semianalytical approaches. Therefore, the computational burden is comparatively heavy. Also, semianalytical methods will provide the needed benchmark solutions with minimal computational cost, to judge the accuracies of the many fully numerical methods 
using spatial discretization based on simple polynomials locally.

In this paper a simply supported rectangular plate with initial imperfections, under a combination of in-plane and out of plane loads, is analyzed by the global Galerkin method. The present method is applied directly to the von Kármán equations to derive a system of cubic order fully coupled NAEs with as many unknowns as desired. Then the resulting system of NAEs is solved by an algebraic equation solver, such as the Newton-Raphson method. Previously, Dai, Paik and Atluri [Dai et al. 2011a] applied the global Galerkin method to the von Kármán plate, and derived the Galerkin-resulting system of NAEs explicitly. However, the explicit expression for Jacobian matrix (the so-called "tangent-stiffness" matrix) was not obtained, and should be calculated symbolically at each step. As a contribution of the present study, we derive the explicit expression of the Jacobian matrix (or the tangent stiffness matrix) of the resulting NAEs, so that the iterative methods, which require the numerical inversion of the Jacobian matrix, may be applied more efficiently. Eliminating the symbolic operations makes the computational efficiency much more improved than that in [Dai et al. 2011a], as will be verified in numerical illustrations.

The global Galerkin method is compared with the incremental Galerkin method, the perturbation method, the finite element method and the finite difference method in solving the von Kármán plate equations under a combination of in-plane and out-of-plane loads to test its accuracy and efficiency. In addition, the buckling behavior and jump phenomenon of the plate are detected and analyzed numerically.

Another topic of this study is to review a recently proposed class of scalar homotopy methods for solving NAEs. Conventionally, the Newton-Raphson method is popularly used to find successively better approximations to the solutions of a real valued nonlinear system. The Newton-Raphson method converges remarkably quickly provided that the initial guess is sufficiently close to the solution. However, the Newton-Raphson method in general requires the inversion of the Jacobian matrix in each iterative step, it is sensitive to the initial guess, and the accuracy of the solution cannot be guaranteed if nearly singular or ill-conditioned Jacobian matrix is encountered. When the Jacobian (tangent stiffness) matrix becomes singular, as in limit load problems, researchers over the past three decades have devised enhancements to the Newton-Raphson method, such as the arclength method. In this paper we present more elegant algorithms which do not involve the inversion of the Jacobian and which are simpler to use when the Jacobian is nearly singular.

We introduce a series of scalar homotopy methods based on the Newton scalar homotopy function. The general form of the Newton homotopy methods is used to incorporate all the existing homotopy methods in a uniform framework. Besides, the present paper provides a concise and clear interpretation for the scalar homotopy methods, and the efficiencies of the various methods are tested through using them to solve the Galerkin-resulting system of NAEs. The presented scalar homotopy methods overcome the several known drawbacks of the Newton-Raphson method:

(1) They can be applied more efficiently than the Newton-Raphson method, when the unknown vector to be solved from the NAEs tends to be large (even through we have limited our study in this paper to only 40 nonlinear algebraic equations).

(2) They completely avoid the need for the inversion of the Jacobian matrix either numerically or analytically (which is impossible in most cases).

(3) They perform much better than the Newton-Raphson method, when the Jacobian matrix is nearly singular, or is severely ill-conditioned. 


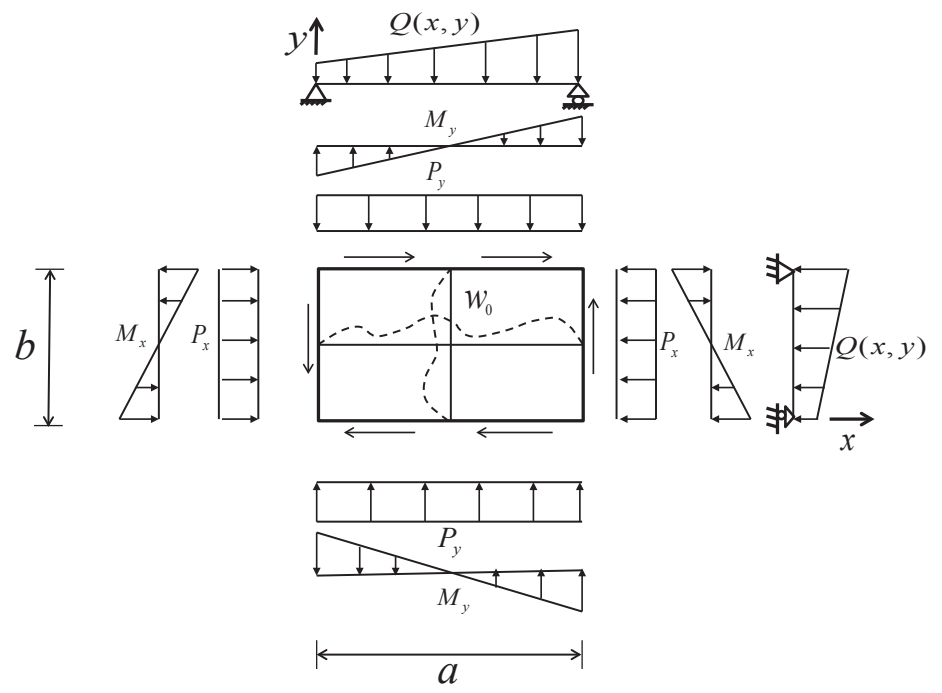

Figure 1. A rectangular plate with initial deflection and general in-plane and lateral loads.

(4) They are insensitive to the guess of the initial solution vector, unlike the Newton-Raphson method.

(5) They can solve either over-determined or under-determined systems of NAEs.

The paper is organized as follows. In Section 2, the global Galerkin method is used to transform the governing PDEs into a system of NAEs. The explicit form of the Jacobian matrix of the NAEs is also derived. In Section 3, various NAE solvers are introduced. A series of Newton homotopy methods are illustrated and classified into continuous-type Newton homotopy and iterative-type Newton homotopy methods. Consequently all the existing Newton homotopy methods are incorporated into a corresponding uniform framework. Moreover, features of the solvers are discussed. We note that Section 3 can stand alone for researchers who are interested in the new NAE solvers. Researchers who focus on the semianalytical methods or nonlinear behavior of the plate may skip this part. Numerical experiments are carried out in Section 4. Finally, we draw some conclusions about the present global Galerkin method and the NAE solvers in Section 5.

\section{Governing equations and the Galerkin method}

The elastic large deflection response of a plate with initial deflection is governed by two PDEs, which are named von Kármán equations. One of them represents the equilibrium condition in the transverse direction, and the other represents the compatibility condition of in-plane strains. The PDEs are as follows:

$$
\begin{gathered}
\phi \equiv D \nabla^{4} w-t\left[\varphi_{, y y}\left(w+w_{0}\right)_{, x x}+\varphi_{, x x}\left(w+w_{0}\right)_{, y y}-2 \varphi_{, x y}\left(w+w_{0}\right)_{, x y}\right]-Q=0, \\
\nabla^{4} \varphi=E\left[w_{, x y}^{2}-w_{, x x} w_{, y y}+2 w_{0, x y} w_{, x y}-w_{0, x x} w_{, y y}-w_{0, y y} w_{, x x}\right] .
\end{gathered}
$$

In the above, $w_{0}$ is the given initial transverse displacement; $w$ is the additional transverse displacement; $Q$ is the lateral pressure acting on the plate; $\varphi$ is the Airy stress function governing the in plane stress 
resultants and $t$ is the plate thickness.

$$
\nabla^{4}=\frac{\partial^{4}}{\partial x^{4}}+2 \frac{\partial^{4}}{\partial x^{2} \partial y^{2}}+\frac{\partial^{4}}{\partial y^{4}}, \quad D=\frac{E t^{3}}{12(1-v)^{2}},
$$

where $D$ is the flexural rigidity, and $\nabla^{4}$ is the well known biharmonic operator; $E$ and $v$ are Young's modulus and Poisson's ratio. The subscripts ${ }_{, x}$ and,$y$ stand for $\partial / \partial x$ and $\partial / \partial y$.

Stress $\sigma_{x}$ in the $x$ direction, $\sigma_{y}$ in the $y$ direction and shear stress $\tau_{x y}$ in $x y$ plane may be expressed as

$$
\sigma_{x}=\varphi_{, y y}, \quad \sigma_{y}=\varphi_{, x x}, \quad \tau_{x y}=\varphi_{, x y} .
$$

We emphasize that, in using the Galerkin method, we need to solve the Airy stress function $\varphi$ first from the (1b), and then apply the Galerkin approach to (1a), which is different from the Rayleigh-Ritz method where the airy stress function $\varphi$ and even the governing equations are not required. RayleighRitz method based on Lagrangian equations is simple in application but computationally expensive since more freedoms are expected by Rayleigh-Ritz method than by Galerkin method, because the deflections of all three directions are required to be assumed. In this study, we derive the explicit expressions of the $\varphi$ and then the resulting NAEs, so that researchers can avoid lengthy algebra and enjoy the advantage of the Galerkin method. The geometry and general loading conditions of the plate is plotted in Figure 1.

In solving the governing equations, the added deflection $w$ due to the applied load, and the initial deflection $w_{0}$ should satisfy the boundary conditions at four edges. All four edges are assumed to be simply supported, and the boundary conditions of the plate are

$$
\begin{aligned}
& w=0, \quad w_{, y y}+v w_{, x x}=0, \quad \text { at } y=0 \text { and } y=b, \\
& w=0, \quad w_{, x x}+v w_{, y y}=0, \quad \text { at } x=0 \text { and } x=a .
\end{aligned}
$$

2.1. Application of the Galerkin method. To satisfy the boundary conditions, the initial deflection $w_{0}$ and the added deflection function $w$ can be normally assumed in double Fourier series,

$$
\begin{gathered}
w_{0}=\sum_{m=1}^{M} \sum_{n=1}^{N} A_{0 m n} \sin \frac{m \pi x}{a} \sin \frac{n \pi y}{b}, \\
w=\sum_{m=1}^{M} \sum_{n=1}^{N} A_{m n} \sin \frac{m \pi x}{a} \sin \frac{n \pi y}{b},
\end{gathered}
$$

where $A_{0 m n}$ and $A_{m n}$ are the known and unknown coefficients, respectively. The present simply supported plate can be solved with various patterns of external loads. The conditions of the combined loads, namely, biaxial loads, biaxial in-plane bending and edge shear are given as follows:

$$
\begin{aligned}
& \int_{0}^{b} \varphi_{, y y} t d y=P_{x}, \quad \int_{0}^{b} \varphi_{, y y} t\left(y-\frac{b}{2}\right) d y=M_{x} \quad \text { at } x=0 \text { and } x=a, \\
& \int_{0}^{a} \varphi_{, x x} t d x=P_{y}, \quad \int_{0}^{a} \varphi_{, x x} t\left(x-\frac{a}{2}\right) d x=M_{y} \quad \text { at } y=0 \text { and } y=b \text {, } \\
& \varphi_{, x y}=-\tau \quad \text { at all four edges. }
\end{aligned}
$$


Then the homogenous solution $\varphi_{h}$ for the Airy stress function $\varphi$ should satisfy the condition of the combined loads acting on the plate. Considering the loading conditions, we can easily find $\varphi_{h}$, by assuming $\varphi_{h}$ as cubic polynomials in $x$ and $y$. Substituting $\varphi_{h}$ into (5) we can obtain,

$$
\varphi_{h}=-P_{x} \frac{y^{2}}{2 b t}-\sigma_{r x} \frac{y^{2}}{2}-P_{y} \frac{x^{2}}{2 a t}-\sigma_{r y} \frac{x^{2}}{2}-M_{x} \frac{y^{2}(2 y-3 b)}{b^{3} t}-M_{y} \frac{x^{2}(2 x-3 a)}{a^{3} t}-\tau_{x y} x y .
$$

The following notations are introduced to abbreviate the expressions involving the sine or cosine terms:

$$
\sin \frac{m \pi x}{a} \equiv s x(m), \quad \cos \frac{m \pi x}{a} \equiv c x(m), \quad \sin \frac{n \pi y}{b} \equiv s y(n), \quad \cos \frac{n \pi y}{b} \equiv c y(n) .
$$

To find the particular solution $\varphi_{p}$ that should satisfy (1b), one ought to substitute $w$ and $w_{0}$ into the right side of (1b), thus obtaining

$$
\begin{aligned}
\nabla^{4} \varphi_{p}=\frac{E \pi^{4}}{4 a^{2} b^{2}} \sum_{m=1}^{M} \sum_{n=1}^{N} \sum_{k=1}^{K} \sum_{l=1}^{L} & \left\{\left[A_{m n} A_{k l} m l(n k-m l)-A_{k l} A_{0 m n}(n k-m l)^{2}\right] c x(m+k) c y(n+l)\right. \\
+ & {\left[A_{m n} A_{k l} m l(n k+m l)+A_{k l} A_{0 m n}(n k+m l)^{2}\right] c x(m+k) c y(n-l) } \\
+ & {\left[A_{m n} A_{k l} m l(n k+m l)+A_{k l} A_{0 m n}(n k+m l)^{2}\right] c x(m-k) c y(n+l) } \\
+ & {\left.\left[A_{m n} A_{k l} m l(n k-m l)-A_{k l} A_{0 m n}(n k-m l)^{2}\right] c x(m-k) c y(n-l)\right\} . }
\end{aligned}
$$

Consequently, motivated by the form of the right-hand side of (7), the particular solution $\varphi_{p}$ for the Airy stress function is assumed as

$$
\begin{array}{r}
\varphi_{p}=\frac{E \pi^{4}}{4 a^{2} b^{2}} \sum_{m=1}^{M} \sum_{n=1}^{N} \sum_{k=1}^{K} \sum_{l=1}^{L}\left\{B_{1}(m, n, k, l) c x(m+k) c y(n+l)+B_{2}(m, n, k, l) c x(m+k) c y(n-l)\right. \\
\left.+B_{3}(m, n, k, l) c x(m-k) c y(n+l)+B_{4}(m, n, k, l) c x(m-k) c y(n-l)\right\} .
\end{array}
$$

Upon substituting (8) into (1b), the coefficients $B_{i}, i=1,2,3,4$ can be readily calculated; they are not written out for saving space. Then, substituting the $B_{i}$ into (8), we obtain the particular solution $\varphi_{p}$ :

$$
\begin{aligned}
\varphi_{p}=\frac{E \alpha^{2}}{4} \sum_{m=1}^{M} \sum_{n=1}^{N} \sum_{k=1}^{K} \sum_{l=1}^{L}\{ & \frac{A_{m n} A_{k l} m l(n k-m l)-A_{k l} A_{0 m n}(n k-m l)^{2}}{\left[(m+k)^{2}+(n+l)^{2}\right]^{2}} c x(m+k) c y(n+l) \\
& +\frac{A_{m n} A_{k l} m l(n k+m l)+A_{k l} A_{0 m n}(n k+m l)^{2}}{\left[(m+k)^{2}+(n-l)^{2}\right]^{2}} c x(m+k) c y(n-l) \\
& +\frac{A_{m n} A_{k l} m l(n k+m l)+A_{k l} A_{0 m n}(n k+m l)^{2}}{\left[(m-k)^{2}+(n+l)^{2}\right]^{2}} c x(m-k) c y(n+l) \\
& \left.+\frac{A_{m n} A_{k l} m l(n k-m l)-A_{k l} A_{0 m n}(n k-m l)^{2}}{\left[(m-k)^{2}+(n-l)^{2}\right]^{2}} c x(m-k) c y(n-l)\right\} .
\end{aligned}
$$

Then, the Airy stress function $\varphi$ can be expressed as

$$
\varphi=\varphi_{h}+\varphi_{p} .
$$

It is evident from Equations (6), (9) and (10) that $\varphi$ is a second-order function with regard to the unknown deflection coefficients $A_{m n}$. To compute the unknown coefficients, the global Galerkin method 
is applied to the equilibrium (1a):

$$
\iiint_{V} \phi(x, y, z) \operatorname{sx}(i) \operatorname{sy}(j) d x d y d z=0 .
$$

Upon substituting (10) into (1a), and then (1a) to (11) after a lengthy derivation, we obtain a system of third-order (cubic) coupled NAEs, with respect to the unknown coefficients, the explicit expression of the nonlinear system of algebraic equations is

$$
\begin{aligned}
& \sum \sum A_{m n} \cdot D \pi^{4}\left(\frac{m^{2}}{a^{2}}+\frac{n^{2}}{b^{2}}\right)^{2} H_{01}(i, j, m, n) \\
& +\sum \sum \sum \sum \sum \sum A_{m n} A_{k l} A_{r s} \cdot(-t) \frac{E \alpha^{2} \pi^{4}}{4 a^{2} b^{2}}\left(H_{1}+H_{2}+H_{3}+H_{4}-2 H_{9}-2 H_{10}-2 H_{11}-2 H_{12}\right) \\
& +\sum \sum \sum \sum A_{m n} A_{k l} \cdot(-t) \frac{E \alpha^{2} \pi^{4}}{4 a^{2} b^{2}} \sum \sum A_{0 r s}\left(H_{1}+H_{2}+H_{3}+H_{4}-2 H_{9}-2 H_{10}-2 H_{11}-2 H_{12}\right) \\
& +\sum \sum \sum \sum A_{k l} A_{r s} \cdot(-t) \frac{E \alpha^{2} \pi^{4}}{4 a^{2} b^{2}} \sum \sum A_{0 m n}\left(H_{6}+H_{7}-H_{5}-H_{8}+2 H_{13}-2 H_{14}-2 H_{15}+2 H_{16}\right) \\
& +\sum \sum A_{k l} \cdot(-t) \frac{E \alpha^{2} \pi^{4}}{4 a^{2} b^{2}} \sum \sum \sum \sum A_{0 m n} A_{0 r s}\left(H_{6}+H_{7}-H_{5}-H_{8}+2 H_{13}-2 H_{14}-2 H_{15}+2 H_{16}\right) \\
& +\sum \sum A_{m n} \cdot(-t)\left\{\frac{m^{2} \pi^{2}}{a^{2}}\left[\left(\frac{P_{x}}{b t}+\sigma_{r x}-\frac{6}{b^{2} t} M_{x}\right) H_{01}(i, j, m, n)+\frac{12}{b^{3} t} M_{x} H_{03}(i, j, m, n)\right]\right. \\
& +\frac{n^{2} \pi^{2}}{b^{2}}\left[\left(\frac{P_{y}}{a t}+\sigma_{r y}-\frac{6}{a^{2} t} M_{y}\right) H_{01}(i, j, m, n)+\frac{12}{a^{3} t} M_{y} H_{02}(i, j, m, n)\right] \\
& \left.+\frac{2 \tau \pi^{2}}{a b} m n \cdot H_{04}(i, j, m, n)\right\} \\
& +\sum \sum A_{0 m n} \cdot(-t)\left\{\frac{m^{2} \pi^{2}}{a^{2}}\left[\left(\frac{P_{x}}{b t}+\sigma_{r x}-\frac{6}{b^{2} t} M_{x}\right) H_{01}(i, j, m, n)+\frac{12}{b^{3} t} M_{x} H_{03}(i, j, m, n)\right]\right. \\
& +\frac{n^{2} \pi^{2}}{b^{2}}\left[\left(\frac{P_{y}}{a t}+\sigma_{r y}-\frac{6}{a^{2} t} M_{y}\right) H_{01}(i, j, m, n)+\frac{12}{a^{3} t} M_{y} H_{02}(i, j, m, n)\right] \\
& \left.+\frac{2 \tau \pi^{2}}{a b} m n \cdot H_{04}(i, j, m, n)\right\}
\end{aligned}
$$$$
-Q \cdot H_{00}(i, j)=0
$$

where for simplicity the coefficient matrix $H_{1}(i, j, m, n, k, l, r, s)$ is denoted by $H_{1}$ and so forth, and all the summations above are carried out over the dummy indexes $m, n, k, l, r, s$, and $i, j$ are free indexes. All the coefficient matrices can be obtained by performing integration over the whole volume of the plate, whose expressions are provided in the Appendix of [Dai et al. 2011a]. We can write the resulting 
algebraic system (12) neatly in matrix form:

$$
\boldsymbol{K}_{t} \boldsymbol{A}_{t}+\boldsymbol{K}_{s} \boldsymbol{A}_{s}+\boldsymbol{K}_{f} \boldsymbol{A}_{f}+\boldsymbol{C}=\mathbf{0},
$$

where $\boldsymbol{C}$ is a constant column matrix, $\boldsymbol{K}_{f}, \boldsymbol{K}_{s}$ and $\boldsymbol{K}_{t}$ are the first-, second- and third-order coefficient matrices - the dimensions (number of rows $\times$ number of columns) of these matrices being

$$
M N \times(M N)^{3} \text { for } \boldsymbol{K}_{t}, \quad M N \times(M N)^{2} \text { for } \boldsymbol{K}_{s}, \quad M N \times M N \text { for } \boldsymbol{K}_{f}, \quad M N \times 1 \text { for } \boldsymbol{C},
$$

— and where $\boldsymbol{A}_{f}, \boldsymbol{A}_{s}$ and $\boldsymbol{A}_{t}$ are the first-, second- and third-order unknown vectors. ${ }^{1}$

\subsection{An explicit derivation of the Jacobian matrix (tangent stiffness matrix) for the von Kármán plate.} Up to now, the NAEs are obtained, and can be solved by applying NAE solvers. However, in most of the cases, the Jacobian matrix of the derived system of NAEs is required to enable the iterative procedures in the course of using the algebraic equation solvers. In our previous work, we only derived the explicit form of algebraic system. The Jacobian matrix to this system is not derived explicitly. Therefore, we resort to symbolic operations embedded in the Matlab to calculate the Jacobian matrix at each iteration. Although very accurate solutions were achieved in [Dai et al. 2011a] via this scheme, we admit that the computational efforts are very heavy. In the present work, we derive the explicit form of the Jacobian matrix to eliminate this drawback. It should be emphasized that the explicit form of the Jacobian matrix of the NAEs resulting from the von Kármán plate PDEs is provided for the first time in literature. This Jacobian matrix needs to be inverted in each iterative step in the Newton-Raphson method, but such an inversion is not necessary in any of the scalar homotopy methods presented in this paper.

The resulting algebraic system (13) can be written in a general form as

$$
\boldsymbol{F}(\boldsymbol{A})=\mathbf{0},
$$

or, particularly

$$
F_{i j}\left(A_{p q}\right)=0, \quad i, p=1,2, \ldots, M ; j, q=1,2, \ldots, N .
$$

It should be noted that $F_{i j}$ does not represent a matrix. It is used to simply denote that there are $M \times N$ equations, with the $[(j-1) N+i]$-th equation being denoted by $F_{i j}$. Similarly, $A_{p q}$ represents the $[(q-1) N+p]$-th unknown coefficient.

$$
B_{u, v}=\frac{\partial F_{i j}}{\partial A_{p q}},
$$

where the $B_{u, v}$ is the $u$-th row and $v$-th column of $\boldsymbol{B}$ with $u=(j-1) N+i, v=(q-1) N+p$. There are eight terms in (12), the first six terms are associated with the unknown coefficients and the last two terms are constant with regard to unknowns. The Jacobian matrix $\boldsymbol{B}$ of (12) is derived term by term as follows.

$$
\frac{\partial F_{i j}^{1}}{\partial A_{p q}}=D \pi^{4}\left(\frac{p^{2}}{a^{2}}+\frac{q^{2}}{b^{2}}\right)^{2} H_{01}(i, j, p, q)
$$

\footnotetext{
${ }^{1}$ The second- and third-order unknown vectors are vectors devised in [Dai et al. 2011a; 2011b] for obtaining the matrix equation (13).
} 


$$
\begin{aligned}
\frac{\partial F_{i j}^{2}}{\partial A_{p q}}= & \sum \sum \sum \sum A_{k l} A_{r s} \times(-t) \frac{E \alpha^{2} \pi^{4}}{4 a^{2} b^{2}}\left(H_{1}+H_{2}+H_{3}+H_{4}-2 H_{9}-2 H_{10}-2 H_{11}-2 H_{12}\right) \\
& +\sum \sum \sum \sum A_{m n} A_{r s} \times(-t) \frac{E \alpha^{2} \pi^{4}}{4 a^{2} b^{2}}\left(H_{1}+H_{2}+H_{3}+H_{4}-2 H_{9}-2 H_{10}-2 H_{11}-2 H_{12}\right) \\
& +\sum \sum \sum \sum A_{m n} A_{k l} \times(-t) \frac{E \alpha^{2} \pi^{4}}{4 a^{2} b^{2}}\left(H_{1}+H_{2}+H_{3}+H_{4}-2 H_{9}-2 H_{10}-2 H_{11}-2 H_{12}\right)
\end{aligned}
$$

the matrices $H_{i}$ in the first, second and third line being evaluated, respectively, as follows:

$$
H(i, j, p, q, k, l, r, s), \quad H(i, j, m, n, p, q, r, s), \quad H(i, j, m, n, k, l, p, q) .
$$

$$
\begin{aligned}
\frac{\partial F_{i j}^{3}}{\partial A_{p q}} & =\sum \sum A_{k l} \times(-t) \frac{E \alpha^{2} \pi^{4}}{4 a^{2} b^{2}} \sum \sum A_{0 r s}\left(H_{1}+H_{2}+H_{3}+H_{4}-2 H_{9}-2 H_{10}-2 H_{11}-2 H_{12}\right) \\
& +\sum \sum A_{m n} \times(-t) \frac{E \alpha^{2} \pi^{4}}{4 a^{2} b^{2}} \sum \sum A_{0 r s}\left(H_{1}+H_{2}+H_{3}+H_{4}-2 H_{9}-2 H_{10}-2 H_{11}-2 H_{12}\right),
\end{aligned}
$$

where the matrices $H_{i}$ in the first and second lines are $H(i, j, p, q, k, l, r, s)$ and $H(i, j, m, n, p, q, r, s)$, respectively.

$$
\begin{aligned}
\frac{\partial F_{i j}^{4}}{\partial A_{p q}} & =\sum \sum A_{r s} \times(-t) \frac{E \alpha^{2} \pi^{4}}{4 a^{2} b^{2}} \sum \sum A_{0 m n}\left(H_{6}+H_{7}-H_{5}-H_{8}+2 H_{13}-2 H_{14}-2 H_{15}+2 H_{16}\right) \\
& +\sum \sum A_{k l} \times(-t) \frac{E \alpha^{2} \pi^{4}}{4 a^{2} b^{2}} \sum \sum A_{0 m n}\left(H_{6}+H_{7}-H_{5}-H_{8}+2 H_{13}-2 H_{14}-2 H_{15}+2 H_{16}\right),
\end{aligned}
$$

where the matrices $H \mathrm{~s}$ in the first and second line are $H(i, j, m, n, p, q, r, s)$ and $H(i, j, m, n, k, l, p, q)$, respectively.

$$
\begin{aligned}
& \frac{\partial F_{i j}^{5}}{\partial A_{p q}}=(-t) \frac{E \alpha^{2} \pi^{4}}{4 a^{2} b^{2}} \sum \sum \sum \sum A_{0 m n} A_{0 r s}\left(H_{6}+H_{7}-H_{5}-H_{8}+2 H_{13}-2 H_{14}-2 H_{15}+2 H_{16}\right) . \\
& \frac{\partial F_{i j}^{6}}{\partial A_{p q}}=(-t)\left\{\frac{p^{2} \pi^{2}}{a^{2}}\left[\left(\frac{P_{x}}{b t}+\sigma_{r x}-\frac{6}{b^{2} t} M_{x}\right) H_{01}+\frac{12}{b^{3} t} M_{x} H_{03}\right]\right. \\
& \left.+\frac{q^{2} \pi^{2}}{b^{2}}\left[\left(\frac{P_{y}}{a t}+\sigma_{r y}-\frac{6}{a^{2} t} M_{y}\right) H_{01}+\frac{12}{a^{3} t} M_{y} H_{02}\right]+\frac{2 \tau \pi^{2}}{a b} p q H_{04}\right\},
\end{aligned}
$$

where the matrices depend on $(i, j, p, q)$. Therefore, the Jacobian matrix is

$$
B_{u, v}=\frac{\partial F_{i j}}{\partial A_{p q}}=\sum_{k=1}^{6} \frac{\partial F_{i j}^{k}}{\partial A_{p q}} .
$$

Consequently, with the explicit form of the system of NAEs and its Jacobian matrix, the present problem can be solved readily by using various NAE solvers. In the course of solving a system of NAEs, the Jacobian matrix is usually necessary. Normally, the numerical approximation of the Jacobian matrix is calculated in each iteration step via numerical difference techniques. The explicitly derived Jacobian 
matrix may significantly accelerate the computing rate of the algebraic solver [Dai et al. 2014a]. The effect of using the explicitly derived Jacobian matrix rather than the numerically calculated one on the computational efficiency has been intensively analyzed in [Dai et al. 2014b] in a two-degree-of-freedom airfoil problem. It was demonstrated that using the explicit Jacobian matrix can be roughly two orders of magnitude faster.

\section{Methods for nonlinear algebraic equations}

The numerical solution of linear or nonlinear, well-conditioned or ill-conditioned, and underdetermined or overdetermined algebraic equations is one of the main aspects of computational mechanics. In many practical nonlinear engineering problems, methods such as the finite element method, boundary element method, finite volume method, the meshless method, global Galerkin method, Rayleigh-Ritz method, etc., eventually lead to a system of nonlinear algebraic equations (NAEs). Many numerical methods used in computational mechanics, as illustrated in [Atluri 2005] lead to the solution of a system of linear algebraic equations for a linear problem, and of a system of NAEs for a nonlinear problem.

A system of nonlinear algebraic equations is

$$
F_{i}\left(x_{j}\right)=0, \quad i, j=1,2, \ldots, n .
$$

Solvers for this set of NAEs are introduced below. In the section of numerical experiments, they are applied to solve the resulting algebraic system from the implementation of the Galerkin method to von Kármán plate equations.

3.1. Newton method and preliminary work. The most famous method for solving nonlinear algebraic equations is the Newton-Raphson method, or Newton method, which is given algorithmically as

$$
\boldsymbol{x}_{k+1}=\boldsymbol{x}_{k}-\boldsymbol{B}_{k}^{-1} \boldsymbol{F}_{k},
$$

where we use $\boldsymbol{x}:=x_{1}, x_{2}, \ldots, x_{n}$ and $\boldsymbol{F}:=F_{1}, F_{2}, \ldots, F_{n}$ to represent the vectors, $\boldsymbol{B}$ is the $n \times n$ Jacobian matrix with its $(i, j)$ entry given by $\partial F_{i} / \partial x_{j}$, and $\boldsymbol{x}_{k+1}$ is the $(k+1)$-th iteration for the unknown vector $\boldsymbol{x}$. Newton method is advantageous in that it converges quadratically fast, provided that the initial "guesses" for the solution are within a certain radius of convergence. However, sometimes Newton method suffers from its sensitiveness to initial "guesses", and the computational burden/accuracy of inverting the Jacobian matrix when the Jacobian matrix is singular or severely ill-conditioned.

Hirsch and Smale [1979] derived a "continuous Newton method" governed by the differential equation

$$
\begin{aligned}
& \dot{\boldsymbol{x}}(t)=-\boldsymbol{B}^{-1} \boldsymbol{F}(\boldsymbol{x}), \\
& \boldsymbol{x}(0)=\boldsymbol{a},
\end{aligned}
$$

where $\boldsymbol{a} \in \mathbb{R}^{n}$. It should be noted that applying a forward Euler scheme to (20) leads to the classical Newton method. Therefore, the continuous Newton method is just the iterative form of Newton method written in the ODE form. The performance does not improve much as compared with the classical Newton method.

Until very recently Newton-type methods are the only choice for solving NAEs, where the inverse of Jacobian matrix is inevitable. To eliminate the need for inverting a matrix in the iteration procedure, a 
straightforward first-order ODE system,

$$
\begin{aligned}
& \dot{\boldsymbol{x}}(t)=-\boldsymbol{F}(\boldsymbol{x}), \\
& \boldsymbol{x}(0)=\boldsymbol{a},
\end{aligned}
$$

was used [Ramm 2004]. However, iteration procedure arising out of the integration of (22) is very sensitive to the initial guess, and converges very slowly. Liu and Atluri [2008] proposed a fictitious time integration method (FTIM) in the form of a system of ODEs as

$$
\dot{\boldsymbol{x}}(t)=-\frac{v}{q(t)} \boldsymbol{F}(\boldsymbol{x}),
$$

where $v$ is a nonzero parameter and $q(t)$ is required to be a monotonically increasing function of $t$. In their approach, the term $v / q(t)$ plays the role of speeding up the convergence. It is noted that an elementary version of the continuation method similar to the FTIM was introduced in [Kane and Levinson 1985].

However, both the methods of FTIM and that in [Ramm 2004] are not rigorously derived mathematically. Interestingly, we can see that both of them do not need the Jacobian matrix, let alone its inversion. However, according to authors' experience, they are extremely sensitive to initial guesses, and converge much more slowly than the Newton method. Therefore both methods are not recommended unless the Jacobian matrix cannot be obtained or involved.

Atluri, Liu and Kuo [Atluri et al. 2009] proposed a modified Newton method (MNM), which is in fact a combination of the continuous Newton method and the FTIM and the finite difference technique, for solving nonlinear algebraic equations avoiding the inverse of the Jacobin matrix. The MNM is given as

$$
\frac{d \boldsymbol{x}_{i}}{d \tau}=-\frac{v}{1+\tau}\left(1-s_{i}\right) \boldsymbol{B}_{i} \frac{\boldsymbol{x}_{i}-\boldsymbol{x}_{i-1}}{\Delta s}+\boldsymbol{F}_{i}=\mathbf{0}, \quad i=1, \ldots, m,
$$

where $s=1-e^{-t}$ is a new variable, and $s \in[0,1)$ is divided into $m$ subintervals with $\Delta s=1 / \mathrm{m}$. Numerical examples of [Atluri et al. 2009] showed that the MNM converges faster than the FTIM in some problems. However, the convergence rate still cannot compare with that of the Newton method. In addition, the ODE system (25) of the MNM is $m$ times larger than the FTIM and the continuous Newton method, which makes the integration much more expensive.

Liu, Yeih, Kuo and Atluri [Liu et al. 2009] developed a scalar homotopy method, which transforms the original NAEs into an equivalent system of ODEs. The scalar homotopy method is totally distinguished from the aforementioned FTIM, MNM methods because the FTIM and MNM are based on (24), which is not a strictly derived relation but rather an intuition.

In solving nonlinear algebraic equations, the homotopy method represents a way to enhance the convergence from a local convergence to a global convergence. Previously, all the homotopy methods are based on the construction of a vector homotopy function, $\boldsymbol{H}(\boldsymbol{x}, t)$ which serves the objective of continuously transforming a function $\boldsymbol{G}(\boldsymbol{x})$ into $\boldsymbol{F}(\boldsymbol{x})$ by introducing a homotopy parameter $t$. The homotopy parameter $t$ can be treated as a time-like fictitious variable, and the homotopy function can be any continuous function such that: $\boldsymbol{H}(\boldsymbol{x}, 0)=\boldsymbol{G}(\boldsymbol{x})$ and $\boldsymbol{H}(\boldsymbol{x}, 1)=\boldsymbol{F}(\boldsymbol{x})$.

Two kinds of homotopy functions are popularly used. The fixed-point homotopy function can be written as

$$
\boldsymbol{H}(\boldsymbol{x}, t)=t \boldsymbol{F}(\boldsymbol{x})+(1-t)\left(\boldsymbol{x}-\boldsymbol{x}_{0}\right)=0,
$$


and the Newton homotopy function is

$$
\boldsymbol{H}(\boldsymbol{x}, t)=t \boldsymbol{F}(\boldsymbol{x})+(1-t)\left[\boldsymbol{F}(\boldsymbol{x})-\boldsymbol{F}\left(\boldsymbol{x}_{0}\right)\right]=0,
$$

where $\boldsymbol{x}_{0}$ is the given initial values and $t \in[0,1]$. Motivated by the above vector homotopy function (26), Liu et al. [2009] proposed a fixed-point scalar homotopy function

$$
h(\boldsymbol{x}, t)=\frac{1}{2} t\|\boldsymbol{F}(\boldsymbol{x})\|^{2}+\frac{1}{2}(t-1)\left\|\boldsymbol{x}-\boldsymbol{x}_{0}\right\|^{2}=0,
$$

Then $\boldsymbol{x}=\boldsymbol{x}(t)$ is assumed in [Liu et al. 2009]. Differentiating (28) on both sides with respect to $t$ yields

$$
\frac{1}{2}\left[\|\boldsymbol{F}(\boldsymbol{x})\|^{2}+\|\boldsymbol{x}-\boldsymbol{a}\|^{2}\right]+\left[t \boldsymbol{B}^{T} \boldsymbol{F}-(1-t)(\boldsymbol{x}-\boldsymbol{a})\right] \cdot \dot{\boldsymbol{x}}=0 .
$$

Also, $\dot{x}$ needs to be parallel to the gradient of the above scalar homotopy function, such that the trajectory of $\boldsymbol{x}$ can be equivalent to seeking of $h(x, t)=0$. Thus,

$$
\dot{\boldsymbol{x}}=-\lambda \frac{\partial h}{\partial \boldsymbol{x}} \text {. }
$$

Therefore, using Equations (28)-(30) the scalar homotopy method (SHM) is derived as

$$
\dot{\boldsymbol{x}}=-\frac{1}{2} \frac{\|\boldsymbol{F}(\boldsymbol{x})\|^{2}+\|\boldsymbol{x}-\boldsymbol{a}\|^{2}}{\left\|t \boldsymbol{B}^{T} \boldsymbol{F}-(1-t)(\boldsymbol{x}-\boldsymbol{a})\right\|^{2}}\left[t \boldsymbol{B}^{T} \boldsymbol{F}-(1-t)(\boldsymbol{x}-\boldsymbol{a})\right] .
$$

The SHM is the first scalar homotopy method, and it is based on the fixed point scalar homotopy function. This method is proved to be less sensitive to initial guess, it has an acceptable convergence rate [Liu et al. 2009]. Systems of over/under determined algebraic equations, or systems being sensitive to initial guesses, or systems whose Jacobian matrix is ill-conditioned, can be solved by the SHM method better than by the Newton method.

3.2. Continuous Newton homotopy methods. In this study, we introduce a series of continuous algorithms based on the Newton homotopy function. The general form of the Newton homotopy methods incorporates all the existing homotopy methods in a uniform framework.

The Newton homotopy function (27) can be written as

$$
\boldsymbol{H}(\boldsymbol{x}, t)=\boldsymbol{F}(\boldsymbol{x})+(t-1) \boldsymbol{F}\left(\boldsymbol{x}_{0}\right)=0 .
$$

Similar to the process in SHM, we can transform the vector Newton homotopy function into a scalar form as follows:

$$
h(\boldsymbol{x}, t)=\frac{1}{2}\|\boldsymbol{F}(\boldsymbol{x})\|^{2}+\frac{1}{2}(t-1)\left\|\boldsymbol{F}\left(\boldsymbol{x}_{0}\right)\right\|^{2}=0 .
$$

Equation (33) holds for all $t \in[0,1]$. To motivate this study, we first consider a fictitious time function $Q(t), t \in[0, \infty)$, where $t$ is the fictitious time and $Q(t)$ has to satisfy that $Q(t)>0, Q(0)=1$, and $Q(t)$ is a monotonically increasing function of $t$, and $Q(\infty)=\infty$. Then we introduce the proposed fictitious time function $Q(t)$ into (33) and have a generalized scalar Newton homotopy function

$$
h(\boldsymbol{x}, t)=\frac{1}{2}\|\boldsymbol{F}(\boldsymbol{x})\|^{2}-\frac{1}{2 Q(t)}\left\|\boldsymbol{F}\left(\boldsymbol{x}_{0}\right)\right\|^{2}=0,
$$


Using the fictitious time function, $Q(t)$, when the fictitious time $t=0$ and $t=\infty$, we can obtain

$$
\begin{aligned}
h(\boldsymbol{x}, t=0) & =\frac{1}{2}\|\boldsymbol{F}(\boldsymbol{x})\|^{2}-\frac{1}{2}\left\|\boldsymbol{F}\left(\boldsymbol{x}_{0}\right)\right\|^{2}=0 \Leftrightarrow \boldsymbol{F}(\boldsymbol{x})=\boldsymbol{F}\left(\boldsymbol{x}_{0}\right), \\
h(\boldsymbol{x}, t=\infty) & =\frac{1}{2}\|\boldsymbol{F}(\boldsymbol{x})\|^{2}=0 \Leftrightarrow \boldsymbol{F}(\boldsymbol{x})=0 .
\end{aligned}
$$

Clearly, the tracking of a solution path for the proposed scalar Newton homotopy function is equivalent to the fictitious time varying from zero to infinity. Multiplying both sides of (34) by $Q(t)$ we have

$$
h(\boldsymbol{x}, t)=\frac{1}{2} Q(t)\|\boldsymbol{F}(\boldsymbol{x})\|^{2}-\frac{1}{2}\left\|\boldsymbol{F}\left(\boldsymbol{x}_{0}\right)\right\|^{2}=0,
$$

We expect $h(\boldsymbol{x}, t)$ to be an invariant manifold in the space of $(\boldsymbol{x}, t)$ for a dynamical system $h(\boldsymbol{x}(t), t)$ to be specified further. With the assumption of $Q(t)>0$, the manifold defined is continuous, and thus the following operation of differential carried out on the manifold makes sense. As a consistency condition, by taking the time differential of (37) with respect to $t$ and considering $\boldsymbol{x}=\boldsymbol{x}(t)$, we have

$$
\frac{1}{2} \dot{Q}(t)\|\boldsymbol{F}(\boldsymbol{x})\|^{2}+Q(t)\left(\boldsymbol{B}^{T} \boldsymbol{F}\right) \cdot \dot{\boldsymbol{x}}=0 .
$$

To transform the original NAEs to ODEs, $\boldsymbol{x}$ should be specified like $\boldsymbol{x}=\lambda \boldsymbol{u}$. It should be emphasized that there are a variety of choices for the form of $\dot{\boldsymbol{x}}=\lambda \boldsymbol{u}$. Various Newton homotopy methods may generate from selections of $\boldsymbol{u}$. Initially, we assume

$$
\dot{\boldsymbol{x}}=\lambda \boldsymbol{u} .
$$

Substituting (39) into (38) yields

$$
\lambda=-\frac{\dot{Q}(t)}{2 Q(t)} \frac{\|\boldsymbol{F}(\boldsymbol{x})\|^{2}}{\boldsymbol{F}^{T} \boldsymbol{B} \boldsymbol{u}},
$$

where, $\lambda$ is a scalar. Plugging $\lambda$ into (39), we have

$$
\dot{\boldsymbol{x}}=-\frac{\dot{Q}(t)}{2 Q(t)} \frac{\|\boldsymbol{F}(\boldsymbol{x})\|^{2}}{\boldsymbol{F}^{T} \boldsymbol{B} \boldsymbol{u}} \boldsymbol{u}
$$

Equation (41) is the general form equation for the continuous Newton homotopy methods. A class of continuous Newton homotopy methods can be obtained from this general equation by reasonably choosing different driving vector $\boldsymbol{u}$. It is found that a fictitious time function is introduced in (41) which is a mathematically equivalent $n$ (if $t$ is implicit) or $n+1$ (if $t$ is explicit) dimensional dynamical system to the original algebraic equation system. The solution for the original algebraic equation can be obtained by applying numerical integration to the equivalent dynamical ODEs.

The fictitious time function $Q(t)$ should be specified before applying the numerical integration. $Q(t)$, as discussed above, should be a monotonically increasing function of $t$. There are many choices for $Q(t)$. According to [Ku et al. 2009], we can choose

so that

$$
Q(t)=e^{\frac{v}{1-m}\left[(1+t)^{1-m}-1\right]},
$$

$$
\frac{\dot{Q}(t)}{Q(t)}=\frac{v}{(1+t)^{m}}, \quad 0<m \leq 1
$$

We make (42) the first choice of $Q(t)$. A simpler, intuitive choice of the fictitious time function is 


\begin{tabular}{lcc}
\hline method & driving vector $\boldsymbol{u}$ & $Q(t)$ \\
\hline DNM1 & $\boldsymbol{B}^{-1} \boldsymbol{F}$ & choice 1 \\
DNM2 & $\boldsymbol{B}^{-1} \boldsymbol{F}$ & choice 2* \\
MBECA1 & $\boldsymbol{B}^{T} \boldsymbol{F}$ & choice 1 \\
MBECA2 & $\boldsymbol{B}^{T} \boldsymbol{F}$ & choice 2 \\
DJIFM1 & $\boldsymbol{F}$ & choice 1 \\
DJIFM2 & $\boldsymbol{F}$ & choice 2 \\
\hline
\end{tabular}

Table 1. A summary of continuous Newton homotopy methods.

$$
Q(t)=e^{t}
$$

which makes $\dot{Q}(t) / Q(t)=1$. This is labeled as the second choice of $Q(t)$.

Finally the general form continuous Newton homotopy method has been derived as (41) with a specified $Q(t)$ in (42) or (44). Integrating this system of ODEs, one can arrive at the stable solution of the ODEs, which is the solution of the original nonlinear algebraic system.

Different choices of the driving vector $\boldsymbol{u}$ in the general Equation (41) lead to different kinds of continuous Newton homotopy methods. See Table 1 for a summary of methods.

Interestingly, if we choose $Q(t)=e^{2 t}$, that is, choice $2^{*}$, instead of $Q=e^{t}$ for the DNM2, we obtain

$$
\dot{\boldsymbol{x}}=-\boldsymbol{B}^{-1} \boldsymbol{F}
$$

which turns out to be exactly the continuous Newton method by Hirsch and Smale [1979]. Applying the forward Euler scheme to (45), we have

$$
\boldsymbol{x}_{k+1}=\boldsymbol{x}_{k}-\boldsymbol{B}_{k}^{-1} \boldsymbol{F}_{k}
$$

which is the classical Newton method.

It can be seen from Table 1 that DNMs are different from the other continuous Newton homotopy methods in that the inverse of the Jacobian matrix is involved. The DNM2 and DNM1 should be regarded as the Newton method and a variant Newton method respectively. However, the DNMs [Ku et al. 2011; $\mathrm{Ku}$ and Yeih 2012] are more flexible than the Newton method, since the dynamical system of the DNMs can be with different choices of $Q(t)$ and numerical integration methods, while the Newton method is a special case with $Q(t)=e^{2 t}$ and the forward Euler method. It is expected that proper selections of $Q(t)$ and integration method may improve the convergence performance.

The MBECAs and the DJIFMs do not involve the inversion of Jacobian matrix. The MBECA1 turns out to be exactly the same as the ECSHA, which is applied in [Dai et al. 2011a].

There are three types of continuous Newton homotopy methods as introduced above. All the three methods are based on the driving vector $\boldsymbol{u}$ where there is only one vector in $\boldsymbol{u}$. To be extended, we can assume $\boldsymbol{u}$ to be constructed by two vectors, such as $\boldsymbol{F}$ and $\boldsymbol{B}^{T} \boldsymbol{F}$, or $\boldsymbol{F}$ and its normal vector $\boldsymbol{P}$, or $\boldsymbol{B}^{T} \boldsymbol{F}$ and its normal vector $\boldsymbol{P}^{*}$. To derive the new methods, the only thing to do is to replace the $\boldsymbol{u}$ in (41). In this study, unless otherwise specified, we use the forward Euler method to perform the integration for the continuous Newton homotopy methods. The performance of the continuous Newton homotopy methods is tested in numerical examples. 
3.3. Iterative Newton homotopy methods. Subsequently, Liu and his coworkers developed a series of purely iterative Newton homotopy methods, where $Q(t)$ no longer needs to be specified. Similar to the continuous Newton homotopy methods, these iterative Newton homotopy methods can also be incorporated into a uniform framework.

To derive the purely iterative methods, the general equation (41) of the continuous Newton homotopy methods is first discretized into a discrete time dynamics via the forward Euler method:

$$
\boldsymbol{x}(t+\Delta t)=\boldsymbol{x}(t)-\beta \frac{\|\boldsymbol{F}(\boldsymbol{x})\|^{2}}{\boldsymbol{F}^{T} \boldsymbol{B} \boldsymbol{u}} \boldsymbol{u}
$$

where

$$
\beta=q(t) \Delta t \quad \text { and } \quad q(t)=\frac{\dot{Q}(t)}{2 Q(t)} .
$$

Then, we differentiate $\boldsymbol{F}$ with respect to $t$, and obtain

$$
\dot{\boldsymbol{F}}=\boldsymbol{B} \dot{\boldsymbol{x}}=-q(t) \frac{\|\boldsymbol{F}\|^{2}}{\left\|\boldsymbol{B}^{T} \boldsymbol{F}\right\|^{2}} \boldsymbol{A F}
$$

where $\boldsymbol{A}=\boldsymbol{B} \boldsymbol{B}^{T}$. Similarly, we use the forward Euler scheme to integrate (49) and get

$$
\boldsymbol{F}(t+\Delta t)=\boldsymbol{F}(t)-\beta \frac{\|\boldsymbol{F}(\boldsymbol{x})\|^{2}}{\boldsymbol{F}^{T} \boldsymbol{B} \boldsymbol{u}} .
$$

Considering that formula (37) is an invariant manifold in time and letting $C=\frac{1}{2}\left\|\boldsymbol{F}\left(\boldsymbol{x}_{0}\right)\right\|^{2}$, we can get

$$
\begin{aligned}
& \|\boldsymbol{F}(t)\|^{2}=\frac{2 C}{Q(t)}, \\
& \|\boldsymbol{F}(t+\Delta t)\|^{2}=\frac{2 C}{Q(t+\Delta t)},
\end{aligned}
$$

since the defined manifold should be invariant with time. Squaring both sides of (50) and using Equations (51) and (52) we can obtain

$$
\frac{C}{Q(t+\Delta t)}=\frac{C}{Q(t)}-2 \beta \frac{C}{Q(t)}+\beta^{2} \frac{C}{Q(t)} \frac{\|\boldsymbol{F}\|^{2}}{\left(\boldsymbol{F}^{T} \boldsymbol{B} \boldsymbol{u}\right)^{2}}\|\boldsymbol{B} \boldsymbol{u}\|^{2} .
$$

After some simple algebra, the following scalar equation is obtained:

$$
a_{0} \beta^{2}-2 \beta+1-s=0,
$$

where

$$
a_{0}:=\frac{\|\boldsymbol{F}\|^{2}\|\boldsymbol{B} \boldsymbol{u}\|^{2}}{\left\|\boldsymbol{F}^{T} \boldsymbol{B} \boldsymbol{u}\right\|^{2}}, \quad s=\frac{Q(t)}{Q(t+\Delta t)}=\frac{\|\boldsymbol{F}(t+\Delta t)\|^{2}}{\|\boldsymbol{F}(t)\|^{2}} .
$$

It worth noting that $s$ can be used as a quantity to assess the convergence property of the iterative Newton homotopy methods, and $a_{0} \geq 1$ according to the Cauchy-Schwarz inequality

$$
\|\boldsymbol{F} \cdot(\boldsymbol{B} \boldsymbol{u})\| \leq\|\boldsymbol{F}\|\|\boldsymbol{B} \boldsymbol{u}\| .
$$


From (54), we can take the solution of $\beta$ to be

$$
\beta=\frac{1-\sqrt{1-(1-s) a_{0}}}{a_{0}} .
$$

To ensure $1-(1-s) a_{0} \geq 0$, let

$$
\begin{aligned}
& 1-(1-s) a_{0}=\gamma^{2} \geq 0, \\
& s=1-\frac{1-\gamma^{2}}{a_{0}},
\end{aligned}
$$

and from (57) it follows that

$$
\beta=\frac{1-\gamma}{a_{0}} .
$$

From Equations (47), (55) and (60) we can obtain the algorithm

$$
\boldsymbol{x}(t+\Delta t)=\boldsymbol{x}(t)-(1-\gamma) \frac{\boldsymbol{F}^{T} \boldsymbol{B} \boldsymbol{u}}{\|\boldsymbol{B} \boldsymbol{u}\|^{2}} \boldsymbol{u},
$$

where

$$
-1<\gamma<1
$$

is a parameter to be chosen by user. Equation (61) is the general form of the iterative Newton homotopy methods.

Using Equations (55), (59) and (62) we derive that

$$
\frac{\|\boldsymbol{F}(t+\Delta t)\|}{\|\boldsymbol{F}(t)\|}=\sqrt{s}<1,
$$

which means that the residual error is absolutely decreased. This property guarantees that the algorithm in (61) is absolutely convergent to the true solution, and a smaller $s$ implies a faster convergence rate.

Recall that the continuous Newton homotopy methods involve the fictitious time function, and the dynamical system (41) should be integrated in time, step by step. Conversely, the iterative Newton homotopy methods are purely iterative, and do not require a specification of $Q(t)$. Different choices of the driving vector $\boldsymbol{u}$ in the general Equation (61) will lead to different iterative Newton homotopy methods as summarized in Table 2, wherein $\boldsymbol{R}=\boldsymbol{B}^{T} \boldsymbol{F}, \boldsymbol{C}=\boldsymbol{B}^{T} \boldsymbol{B}$.

The RNBA is the first iterative Newton homotopy method, which employs one vector $\boldsymbol{R}$ in the driving vector $\boldsymbol{u}$. Later, a series of iterative Newton homotopy methods employing two vectors in the driving vector are developed. The OVDA uses $\boldsymbol{u}=\alpha \boldsymbol{F}+\beta \boldsymbol{R}$, and the dividing parameters $\alpha$ and $\beta$ are determined by letting $\partial s / \partial \alpha=0$ and $\beta=1-\alpha$.

Liu, Dai and Atluri [2011a; 2011b] proposed the OIA/ODVs and the OIAs. The main difference between the OIA/ODVs, OIAs and the OVDA is that two orthogonal vectors instead of the couple of $\boldsymbol{F}$ and $\boldsymbol{R}$ are used to constitute the driving vector. Numerical examples in [Liu et al. 2011a; 2011b] illustrated that OIA/ODVs and OIAs have a better performance than the OVDA in solving their selected problems, while this is not always the case. Numerical examples of this study indicate that the OIAs and the OVDA are comparable in terms of convergence rate, while the OIA/ODVs converge more slowly. In particular, the OIA/ODV[R] is several times slower than the OIAs and the OVDA. So the OIAs are believed to be superior to the OIA/ODVs. 


\begin{tabular}{lccc}
\hline method & driving vector $\boldsymbol{P}$ & $\boldsymbol{P}$ (or $\left.\boldsymbol{P}^{*}\right)$ & parameter optimization scheme \\
\hline RNBA [Liu and Atluri 2011b] & $\boldsymbol{R}$ & $*$ & $*$ \\
OVDA [Liu and Atluri 2011a] & $\alpha \boldsymbol{F}+\beta \boldsymbol{R}$ & $*$ & $\partial s / \partial \alpha=0, \beta=1-\alpha$ \\
OIA/ODV[R] [Liu et al. 2011a] & $\alpha \boldsymbol{R}+\beta \boldsymbol{P}$ & $\boldsymbol{R}-\frac{\|\boldsymbol{R}\|^{2}}{\boldsymbol{R}^{T} \boldsymbol{C} \boldsymbol{R} \boldsymbol{R}}$ & $\partial s / \partial \alpha=\partial s / \partial \beta=0$ \\
OIA/ODV[F] [Liu et al. 2011a] & $\alpha \boldsymbol{F}+\beta \boldsymbol{P}^{*}$ & $\boldsymbol{F}-\frac{\|\boldsymbol{F}\|^{2}}{\boldsymbol{F}^{\boldsymbol{C} \boldsymbol{F}} \boldsymbol{F}}$ & $\partial s / \partial \alpha=\partial s / \partial \beta=0$ \\
OIA(R) [Liu et al. 2011b] & $\alpha \boldsymbol{R}+\beta \boldsymbol{P}$ & $\boldsymbol{F}-\frac{\boldsymbol{R} \cdot \boldsymbol{F}}{\|\boldsymbol{R}\|^{2}}$ & $\partial s / \partial \alpha=\partial s / \partial \beta=0$ \\
OIA(F) [Liu et al. 2011b] & $\alpha \boldsymbol{F}+\beta \boldsymbol{P}^{*}$ & $\boldsymbol{R}-\frac{\boldsymbol{R} \cdot \boldsymbol{F}}{\|\boldsymbol{F}\|^{2}}$ & $\partial s / \partial \alpha=\partial s / \partial \beta=0$ \\
LOIA [Liu and Atluri 2012] & $\alpha \boldsymbol{F}+\boldsymbol{R}$ & $*$ & $\partial s / \partial \alpha=0, \beta=1-\alpha$ \\
GOIA [Liu and Atluri 2012] & $\alpha \boldsymbol{F}+\boldsymbol{R}$ & $*$ & global minimum of $s$ \\
\hline
\end{tabular}

Table 2. A summary of iterative Newton homotopy methods.

It can be seen from Table 2 that the LOIA is essentially similar to the OVDA method, so only the OVDA is evaluated via numerical experiments. For more details about the Newton homotopy methods, one is recommended to refer to related references in Table 2.

\section{Numerical examples}

In this section, examples concerning the solution of von Kármán nonlinear plate equations, for a plate undergoing various kinds of loads are provided to verify the present method as well as to evaluate the novel algebraic equation solvers. The Young's modulus and Poisson's ratio are $E=205.8 \times 10^{9}$ and $v=0.3$, unless otherwise specified. For NAE solvers, the parameter $\gamma=0.3$ and the stop criterion $\epsilon=10^{-4}$ are fixed throughout the paper.

In some examples, the external loads are applied in terms of critical values $P_{\mathrm{xcr}}$ or $\tau_{\mathrm{cr}}$. The critical values of $P_{\mathrm{xcr}}$ and $\tau_{\mathrm{cr}}$ for a plate depend on its supporting pattern as well as length versus width ratio. For a simply supported plate with $a / b=1$ or 2 , the critical values are given as follows

$$
\begin{aligned}
\sigma_{\mathrm{xcr}} & =4 \times \frac{\pi^{2} D}{b^{2} t}, \quad \frac{a}{b}=1,2, \\
\tau_{\mathrm{cr}} & =k \times \frac{\pi^{2} D}{b^{2} t}, \quad k=9.34 \text { if } \frac{a}{b}=1 ; k=6.6 \text { if } \frac{a}{b}=2,
\end{aligned}
$$

where $\sigma_{\mathrm{xcr}}=P_{\mathrm{xcr}} /(b t)$. For more types of supporting forms and length/width ratios, one may refer to Chapter 9 of [Timoshenko 1961].

4.1. A square plate under uniaxial compression. In this example, a simply supported square plate under uniaxial compression is analyzed. The dimensions of this plate are $a=1, b=1, t=0.009$, where $a, b, t$ represent length, width and thickness respectively. All dimensions in this study are in meters unless 


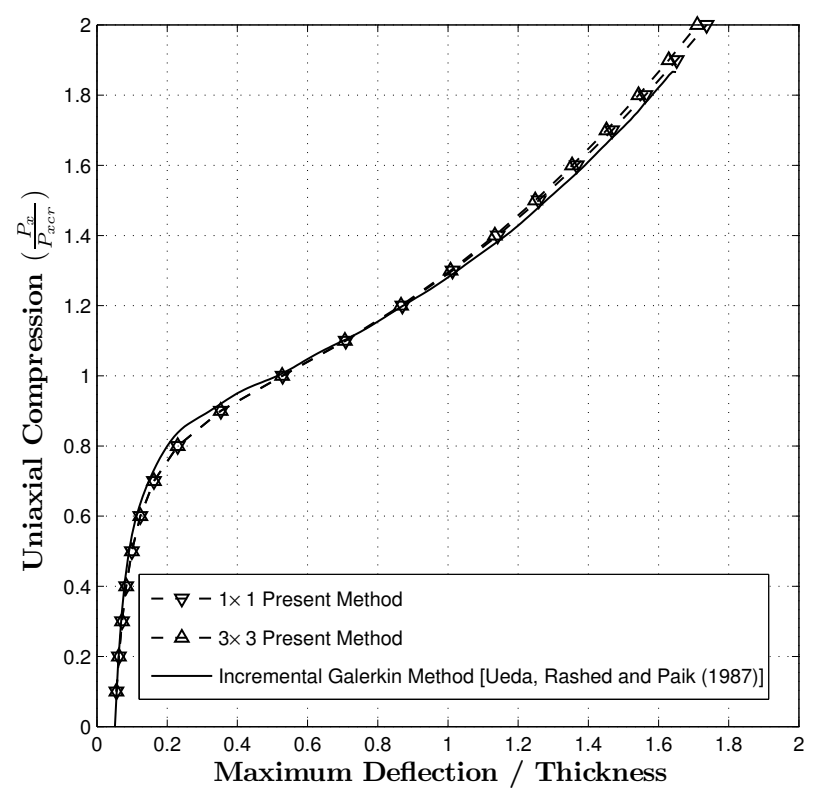

Figure 2. Deflection versus compression loads.

otherwise mentioned. The initial deflection is specified as $A_{0 m n}=0.45 \times 10^{-3} . P_{\text {xcr }}$ is the critical compression causing buckling of plate.

To examine the accuracy of the present global Galerkin method, a case of the incremental Galerkin method developed by Ueda, Rashed and Paik [Ueda et al. 1987] is used to compare with the present method. Figure 2 plots the load-deflection relationships obtained at different load levels. The compression load acting on the plate varies from 0.1 to 2 with load step being 0.1 . Therefore, there are 20 load steps and hence 20 sets of NAEs to solve. For the first load step, the initial values are chosen as a set of small values rather freely, since the expected solution is small. In addition to the first load step, the initial values to start the NAE solver are obtained through a load marching procedure, where the solution of the previous load step is used as the initials of the current load step.

It can be seen from Figure 2 that the results of the present Galerkin method and the incremental Galerkin method are in very good agreement. Figure 2 also reveals that the present method with nine term $(3 \times 3)$ deflection function (labeled as $3 \times 3$ present method) agrees very well with the $1 \times 1$ present method. In the square plate case, $1 \times 1$ present method is quite accurate owing to the similarity between the one half wave plate deflection and the $1 \times 1$ deflection function, which indicates solving the simply supported square plate is extremely economic via the present global Galerkin method. Numerical calculations throughout the paper are performed in Matlab on a personal computer with an Intel core i5 CPU.

4.1.1. Comparative performance of various NAE solvers. Another purpose of the present study is to evaluate the various kinds of NAE solvers. We employ the $3 \times 3$ present method to solve the von Kármán plate problem. Thus, a system of nine NAEs is obtained. Table 3 provides the computational information for solving the resulting system of NAEs via various kinds of solvers. The number of iterations, computing time, and time per iteration (TPI) are listed in Table 3, from which we can see that DNM2, that is, 


\begin{tabular}{lrcc}
\hline method & time(s) & iterations & TPI(s) \\
\hline SHM & 2923.9 & 184679 & 0.0158 \\
MBECA1 & 289.9 & 19144 & 0.0151 \\
MBECA2 & 859.7 & 56758 & 0.0151 \\
DNM1 & 1.9 & 76 & 0.0255 \\
DNM2 & 1.7 & 72 & 0.0233 \\
DJIFM1 & 26.9 & 1849 & 0.0145 \\
DJIFM2 & 51.8 & 3419 & 0.0152 \\
\hline OVDA & 20.2 & 999 & 0.0202 \\
OIA/ODV[R] & 317.4 & 16142 & 0.0197 \\
OIA/ODV[F] & 26.5 & 1341 & 0.0198 \\
OIA(R) & 20.2 & 1020 & 0.0198 \\
OIA(F) & 20.1 & 1001 & 0.0201 \\
GOIA & 19.6 & 980 & 0.0200 \\
\hline
\end{tabular}

Table 3. Example 1: $3 \times 3$ present method.

the classical Newton method, and its variant DNM1 are the fastest ones. It implies that the best driving vector should employ the inverse of the Jacobian matrix. However, since the DNM1 and DNM2 require calculating the inverse of the Jacobian matrix, the TPIs of them are expected to be larger than those of the other methods, which is justified in Table 3. It is emphasized that the larger TPIs do not influence much the computational costs due to the relatively smaller size of the Jacobian matrix. However, when the number of NAEs is very large, the initial "guesses" of solution are not easy to generate, and when the Jacobian matrix is nearly singular or severely ill-conditioned, the advantages of the scalar homotopy methods such as the GOIA which does not need to invert the Jacobian matrix start becoming apparent.

The FTIM and MNM are applied to the present case; however, neither gives a convergent solution. The preliminary SHM based on the fixed-point homotopy function converges several times more slowly than the Newton homotopy methods based on the Newton homotopy function. In general, the number of iterations of the iterative methods of the lower part table is smaller than that of the continuous methods of the upper part table. However the OIA/ODV[R] is verified to be an exceptional case in the family of iterative methods, which costs ten more times iterations than its counterpart OIA/ODV[F]. The iterative Newton homotopy methods cost less iterations than the continuous Newton homotopy methods in general. As shown in Table 3, the OVDA, the OIAs and the GOIA are comparable, and promise to be the best Jacobian-inverse-free methods; they may play an important role in solving a nonlinear problem whose initial guess is hard to choose, when Jacobian matrix is ill-conditioned or nearly singular, when the number of NAEs tends to be very large and when the system of NAEs is overdetermined or underdetermined.

4.2. A rectangular plate under uniaxial compression. A simply supported rectangular plate under uniaxial compression is considered. Its dimensions are $a=1.68, b=0.98$ and $t=0.011$. The initial deflection is given by $A_{011}=1.1 \times 10^{-3}$ and $A_{021}=0.22 \times 10^{-3}$. The present Galerkin method is applied to solve this rectangular plate. Besides, the analysis is also carried out by the FEM using rectangular, 


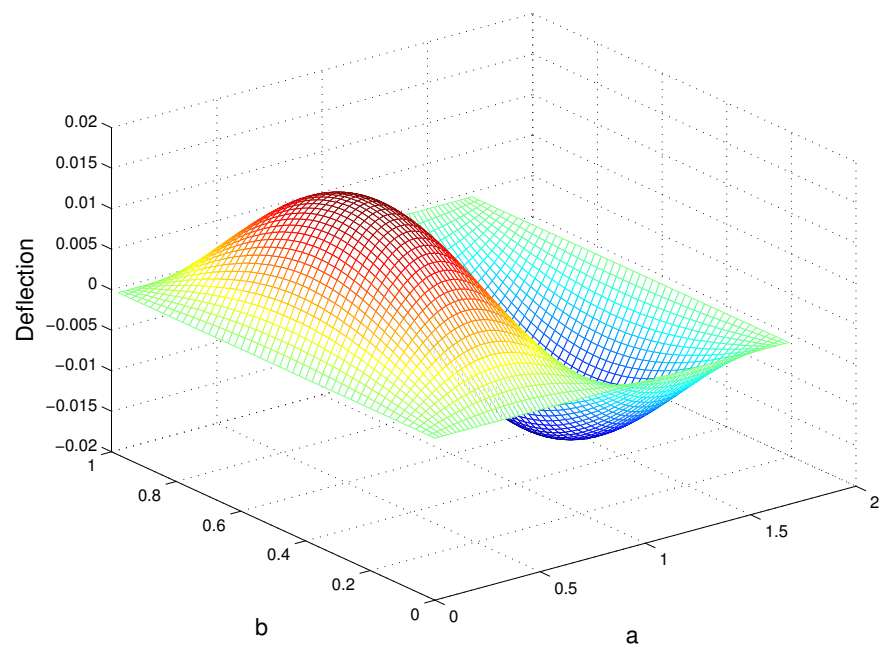

Figure 3. Deformation of a rectangular plate under uniaxial compression: load $=2$.

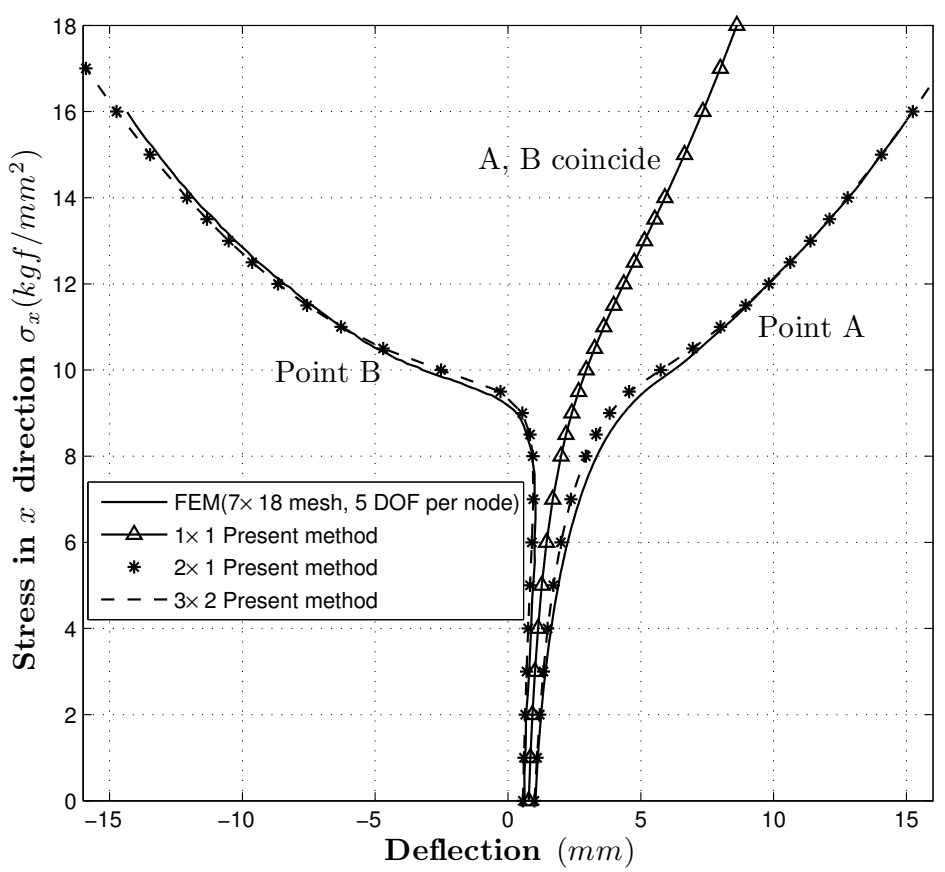

Figure 4. Stress $\sigma_{x}$ versus deflection of point $\mathrm{A}$ and $\mathrm{B}$, for the present Galerkin method and the finite element method.

four node, and nonconforming plate elements with five degrees freedom at each node; $7 \times 18$ elements for half of the plate.

Figure 3 provides the deformation of the rectangular plate. Figure 4 displays curves that plot the stress $\sigma_{x}$ against the deflection of two points $\mathrm{A}(0.25 a, 0.5 b)$ and $\mathrm{B}(0.75 a, 0.5 b)$. It reveals that the results 
of the present method and that of the FEM are in good agreement, which confirms the accuracy of the present method.

Comparing the solutions by the present methods in Figure 4, we see that the global Galerkin method with $1 \times 1$ term deflection function cannot provide an acceptable solution, Physical intuition tells us that the deformation of the plate cannot be described by one term deflection function anyway. It can be seen from Figure 3 that the deformation of the plate has two half waves in the $x$ direction; therefore at least two terms should be used in $x$ direction. The result of $2 \times 1$ present method is in a very good agreement with the result of FEM. Figure 4 also displays that solutions by $3 \times 2$ and $2 \times 1$ present methods are overlapped, which indicates that present method with only a few modes can provide a very accurate solution for the simply supported von Kármán plate under uniaxial compression.

4.2.1. Performance of solvers. The $3 \times 2$ present method is used to solve the rectangular plate, and the computation information for various solvers for solving the Galerkin-resultant NAEs is provided in Table 4.

In accordance with Example 1 (Table 3), the DNM1 and DNM2 involved with the inverse of the Jacobian matrix have larger TPIs, while the total computing time and consumed iterations of the DNMs are much more cheap. The reason is that the usage of $\boldsymbol{B}^{-1}$ provides the best descent direction to reduce residuals (hence requiring fewer iterations), and the time consumption of inverting the current Jacobian matrix is not expensive. Although in the present case the slightly different TPIs are not sufficient to reverse the overall performance of the Newton method and the homotopy methods. It is reasonable to expect that when the size of Jacobian is very large, the Newton method would suffer from a larger TPI. Also, the iterative Newton homotopy methods are computationally cheaper than the continuous-type methods in general, except the OIA/ODV[R].

\begin{tabular}{lcrc}
\hline method & time(s) & iterations & TPI(s) \\
\hline MBECA* & 600.7 & 11208 & 0.0536 \\
MBECA1 & 39.4 & 12555 & 0.0031 \\
MBECA2 & 63.3 & 20537 & 0.0031 \\
DNM1 & 0.51 & 109 & 0.0047 \\
DNM2 & 0.55 & 114 & 0.0048 \\
DJIFM1 & 4.6 & 1424 & 0.0032 \\
DJIFM2 & 5.9 & 1861 & 0.0032 \\
\hline OVDA* & 53.2 & 633 & 0.0840 \\
OVDA & 2.3 & 663 & 0.0035 \\
OIA/ODV[R] & 20.4 & 6336 & 0.0032 \\
OIA/ODV[F] & 2.9 & 866 & 0.0033 \\
OIA(R) & 2.2 & 636 & 0.0034 \\
OIA(F) & 2.3 & 659 & 0.0034 \\
GOIA & 2.3 & 665 & 0.0035 \\
\hline
\end{tabular}

Table 4. Example 2: $3 \times 2$ present method. 


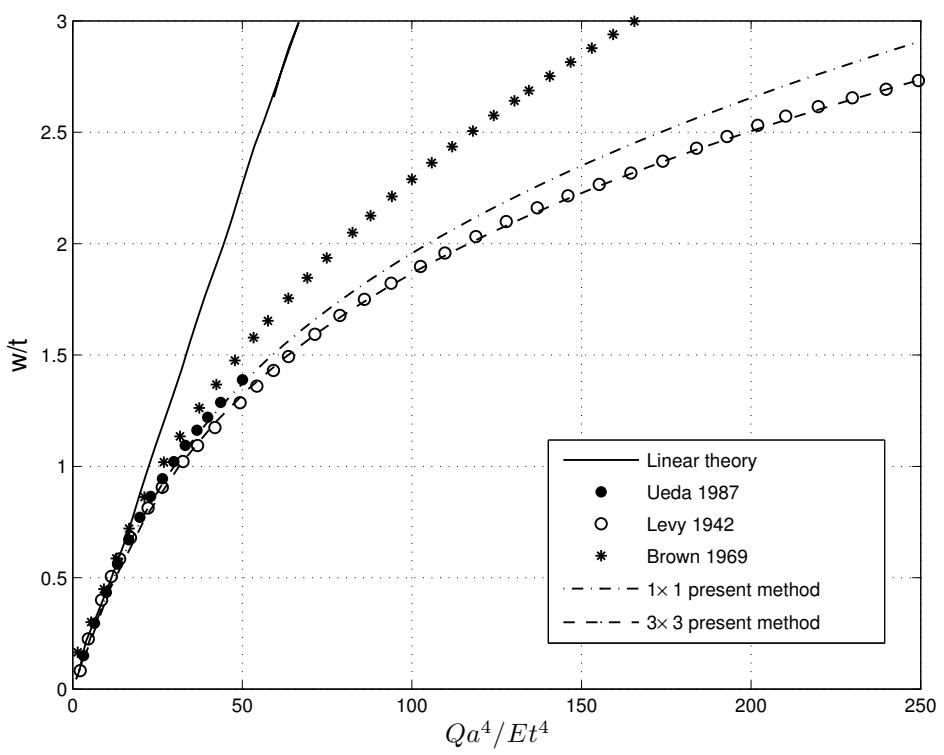

Figure 5. Square plate: central deflection versus lateral pressure.

In addition, the MBECA* [Dai et al. 2011a] (or named ECSHA) and the OVDA* [Dai et al. 2011b] using symbolic calculations of the Jacobian matrix at each iteration are also listed in Table 4 . We see that inclusion of the symbolic operations slightly improves the performance of the solvers in terms of the consumed number of iterations, because using symbolic operations can avoid the cut-off errors which inherently exist in numerical calculations. Nevertheless, the TPIs of MBECA* and OVDA* are about ten times larger than those of the MBECA and OVDA, which indicates that the contribution of deriving the explicit Jacobian matrix is of significant importance. Table 4 shows that the GOIA, the OIAs and the OVDA are the most efficient Jacobian-inverse-free methods. Next to the above three methods are the OIA/ODV $[\mathrm{F}]$ and the DJIFMs; the OIA/ODV[R] and the MBECAs are several orders of magnitude more expensive. It is found that Newton homotopy methods having a driving vector with $\boldsymbol{F}$ inside are superior to those where $\boldsymbol{F}$ is not included.

4.3. A square/rectangular plate subjected to lateral load. A square plate, with geometry $a=b=1$ and $t=0.009$, subjected to a uniformly distributed lateral load $Q$ is considered in this example without initial imperfections.

It indicates in Figure 5 that the present method is quite accurate in solving a plate under lateral load through a comparison with the incremental Galerkin method [Ueda et al. 1987], and Levy's method. The result of $1 \times 1$ present method agrees well with that of $3 \times 3$ present method. We conclude that for a square plate, the present method with very few terms can be reasonably accurate, due to the similarity between the real plate deflection and the one half-wave bulge of the assumed function.

Figure 5 also gives the result via a finite difference method proposed by Brown and Harvey [1969]. It is seen that there is a discrepancy between the present method and the finite difference method. The differences between the solutions, which may be attributed to two different sets of boundary conditions, both of which might be loosely described as simply supported, indicate the importance of specifying 


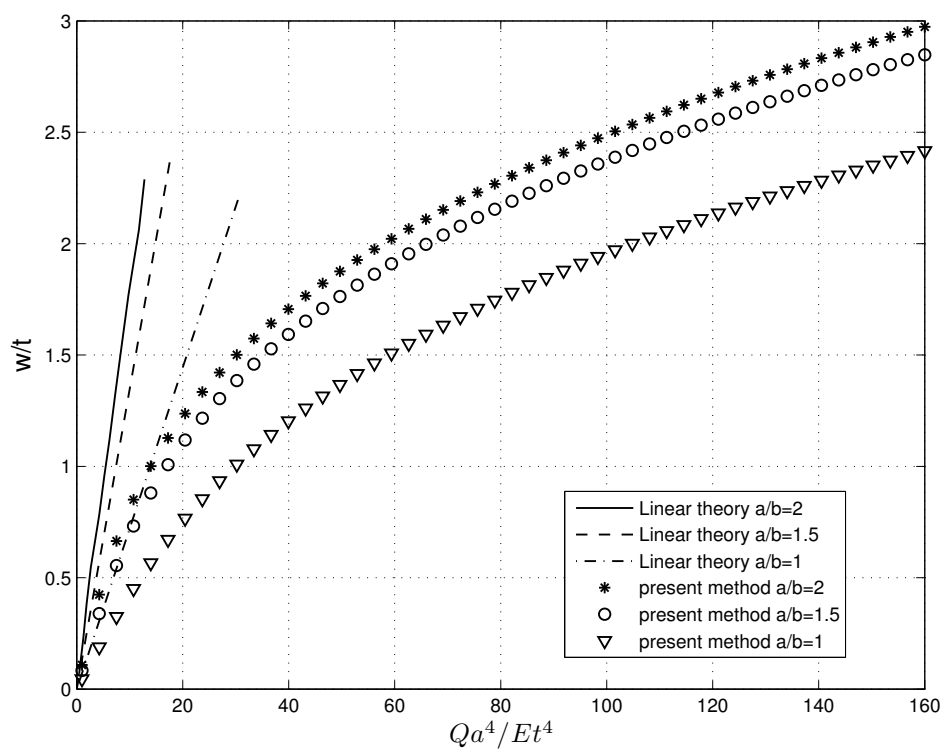

Figure 6. Maximum deflection versus lateral pressure for a range of length/width ratios.

\begin{tabular}{cclcccc}
\hline plate number & $a / b$ & $b / t$ & $E(\mathrm{GPa})$ & $v$ & $Q$ & $P_{x}$ \\
\hline 1 & 1 & 50 & 205.8 & 0.30 & varies & $0.60 P_{\text {xcr }}$ \\
2 & 3 & 66.7 & 204.8 & 0.33 & $29400(\mathrm{~Pa})$ & varies \\
3 & 3 & 66.7 & 204.8 & 0.33 & $49000(\mathrm{~Pa})$ & varies \\
4 & 3 & 74.7 & 214.6 & 0.33 & $1.43\left(\frac{Q b^{4}}{E t^{4}}\right)$ & varies \\
5 & 3 & 74.7 & 214.6 & 0.33 & $4.28\left(\frac{Q b^{4}}{E t^{4}}\right)$ & varies \\
\hline
\end{tabular}

Table 5. Geometries and material properties of plates.

all four boundary conditions. In a further study, we are trying to replace the loose simply supported conditions used in [Levy 1942b; Ueda et al. 1987; Paik et al. 2001; Dai et al. 2011a] by strict simply supported conditions using the present method.

Figure 6 shows the deflection-load relationships for various length/width ratios, and the results by the linear theory are also provided. It can be seen that increasing the ratio of length versus width will lead to a larger plate deflection, which is in accord with physical intuition. Under the same load situation, we expect that an infinite large length/width ratio causes the largest deflection of a plate.

\subsection{A square/rectangular plate subjected to lateral pressure combined with uniaxial compression.} First, a square plate subjected to lateral pressure combined with uniaxial compression is considered. The $x$-direction compression acting on the plate is a constant (see plate 1 in Table 5), and the lateral pressure acting on the plate increases as shown in Figure 7. The geometric and material properties are listed in Table 5, and the initial deflection is zero. For comparison purpose, this plate has also been analyzed via 


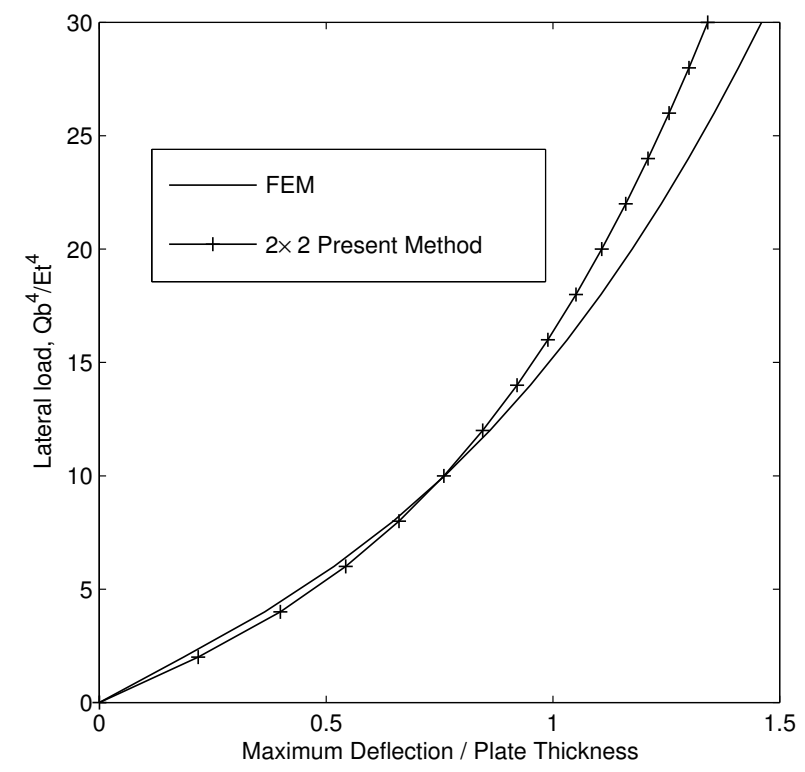

Figure 7. Square plate 1: maximum deflection versus lateral pressure by the present method and the FEM.

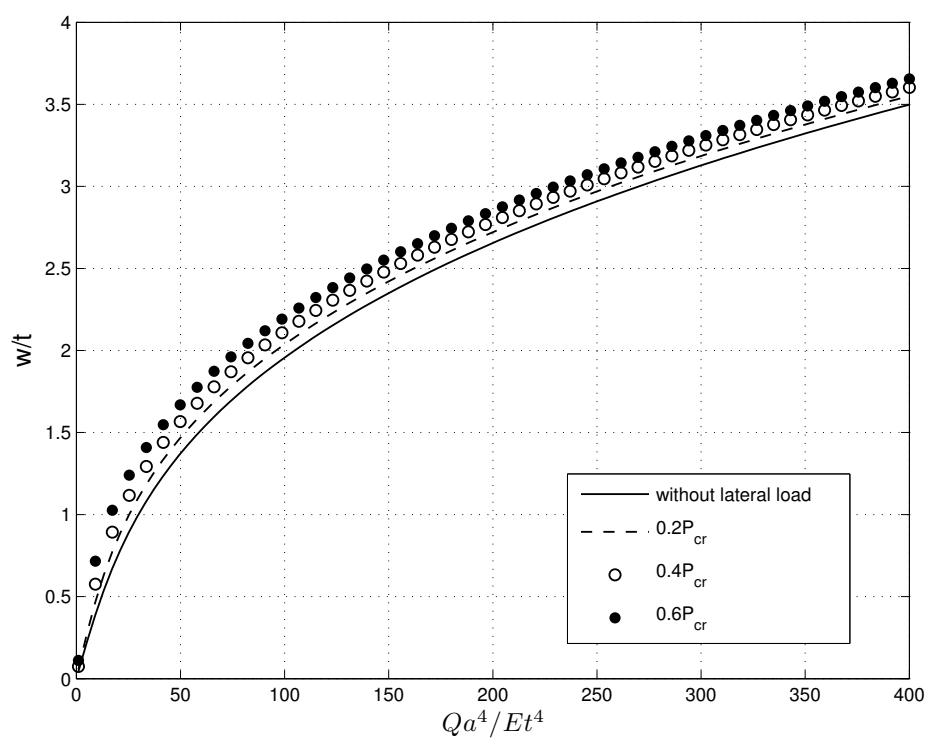

Figure 8. Square plate 1: maximum deflection versus lateral pressure for a range of edge compressions.

FEM in ANSYS (brick element with eight node and three degrees freedom at each node, $50 \times 50 \times 1$ elements for the whole volume).

It is seen from Figure 7 that the results of the present method are quite in accordance with those of the FEM when the lateral pressure is below approximate 15. As the lateral pressure increases further, the discrepancy between the two methods increases. 

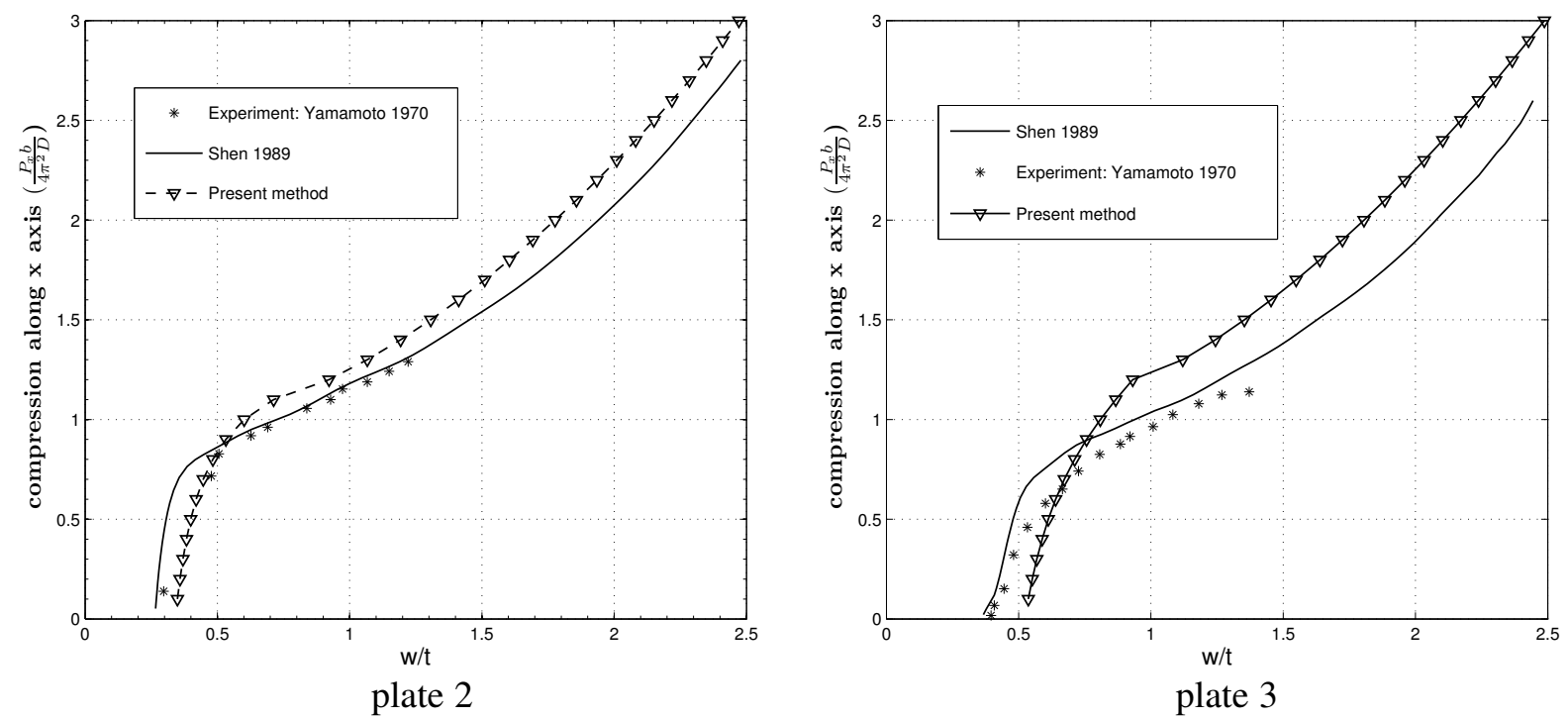

Figure 9. Uniaxial compression versus maximum deflection with a constant lateral pressure by the present method, the perturbation method [Shen 1989] and the experiment [Yamamoto et al. 1970]. Axial load versus deflection: plate 2 (left) and plate 3 (right).

Figure 8 shows the deflection versus lateral pressure curves for square plate 1 under a range of edge compressions. It indicates that as the edge loading increases the plate deflection increases accordingly under the same lateral pressure.

In the first case of plate 1, the axial compression is kept constant and the lateral pressure is increased incrementally. We also analyze the cases, rectangular plates 2-5, wherein the lateral pressure is exerted first and kept constant, and $x$-direction compression is increased gradually.

Figure 9 shows the comparisons of the load-deflection relationships by the present method, the perturbation method [Shen 1989], and the experiment results [Yamamoto et al. 1970]. We see that the present method approximately agrees with the perturbation method and the experiment. The present method is more closer to the experiment than the perturbation method when the compression is relatively low. However, as the load increases, the deflection by the present method is smaller than that of the perturbation method and the experiment. The discrepancy between the present method and the experiment is approximately a constant value, larger than the discrepancy between the perturbation method and the experiment. The present method is simpler than the perturbation method in [Shen 1989]. Because in [Shen 1989], the Galerkin method is used to first convert lateral pressure into an initial deflection, and then governing equations are studied using a perturbation method, taking deflection as its perturbation parameter.

Figure 10 displays the deflection-axial load relations for a range of lateral pressures. The curves are computed through a load-marching procedure wherein the solution of the previous load step is used as the initial of the current load step. In Figure 10, both forward marching and backward marching paths are plotted. It can be seen that with the increase of the lateral pressure, a hysteresis phenomenon may occur. In this case, $Q=3.7$ has no hysteresis while $Q=4$ has hysteresis; so the buckling behavior of 

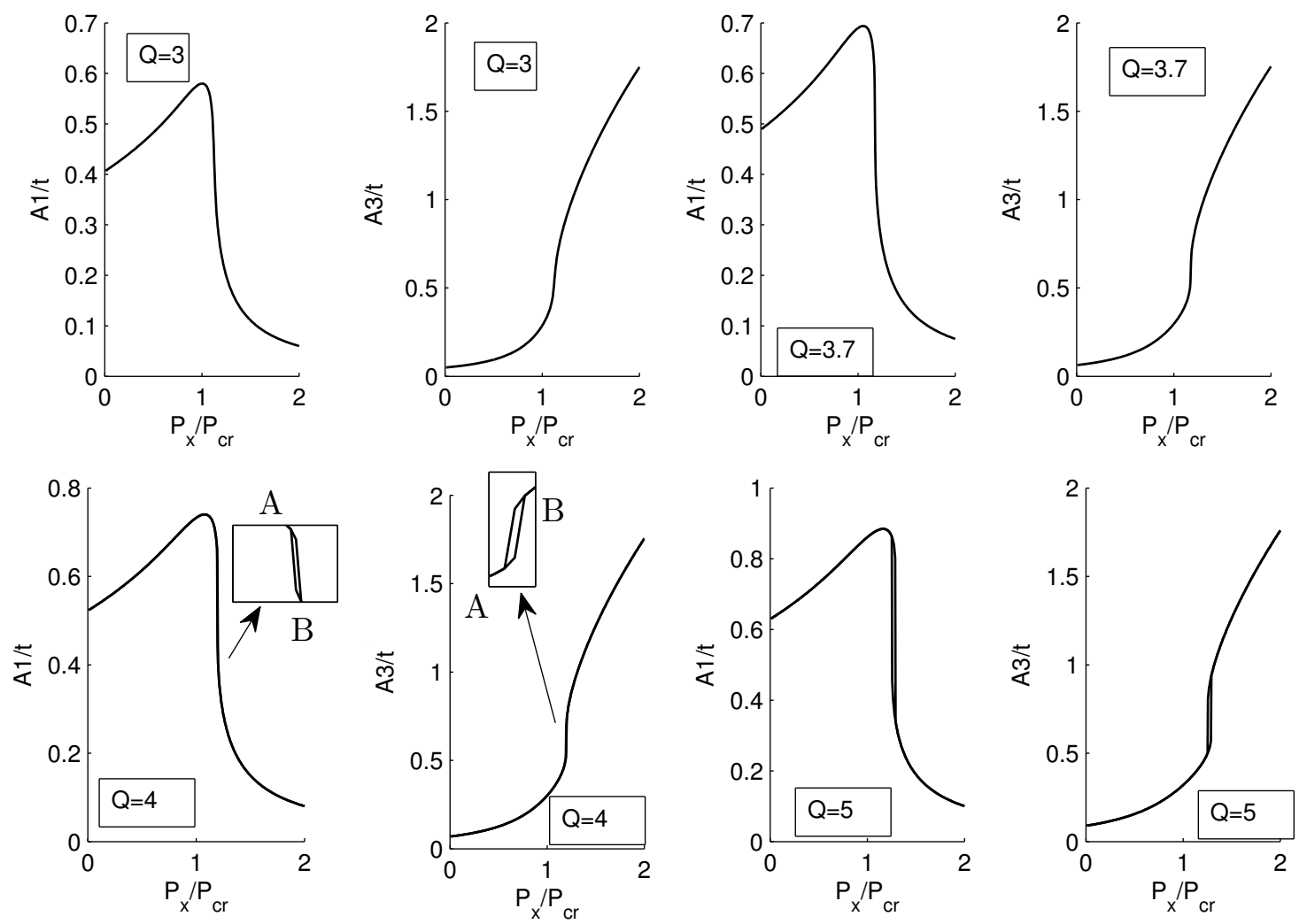

Figure 10. The deflection versus axial load relationships for a range of lateral loading $Q$ s: $Q=3,3.7$, no hysteresis; $Q=4,5$, hysteresis occurs.
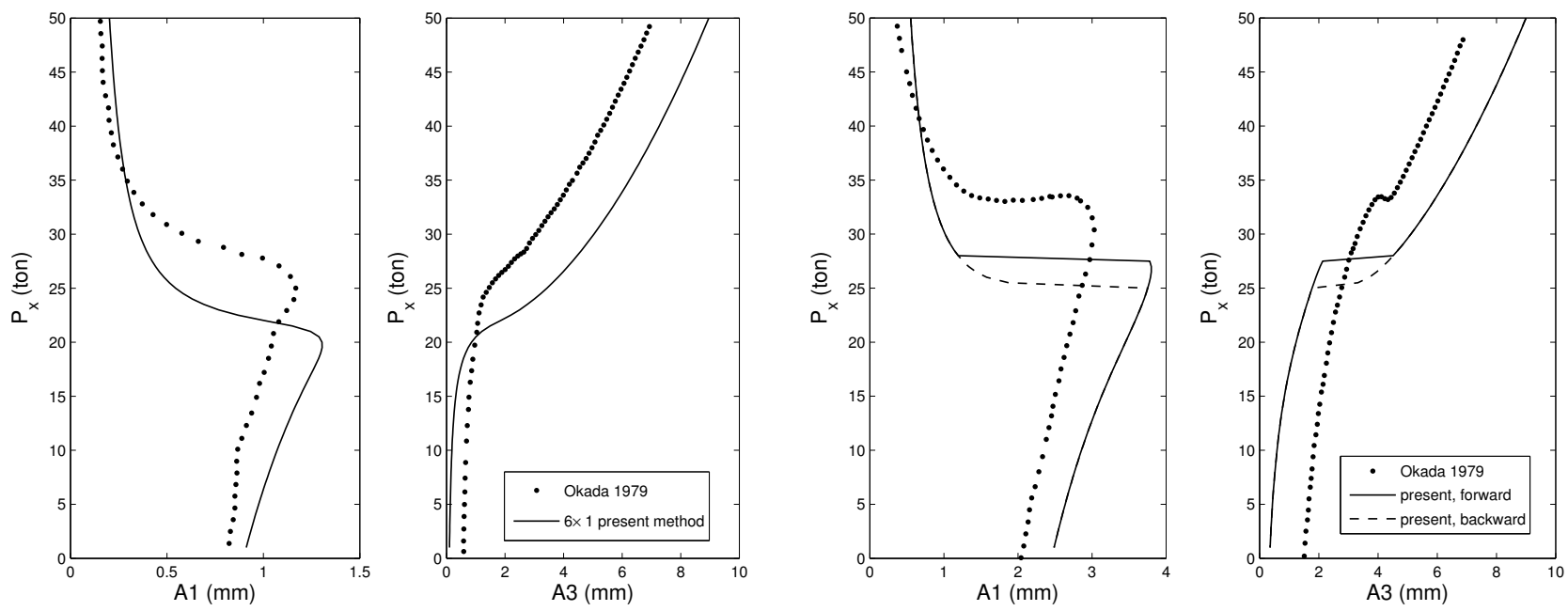

plate 4

plate 5

Figure 11. Axial load versus deflection: comparison between present method and [Okada et al. 1979]: not buckling (plate 4); buckling (plate 5). 


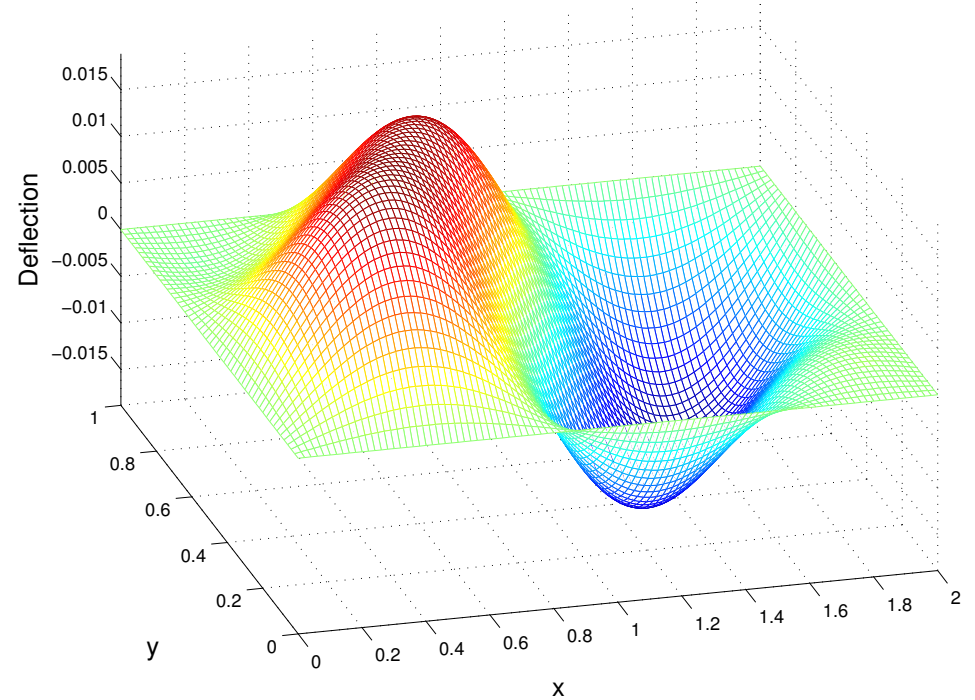

Figure 12. Deformation of a rectangular plate under uniaxial compression $P_{x}=0.65 P_{\mathrm{xcr}}$ (applied first and keep constant) and shear $\tau=2 \tau_{\mathrm{cr}}$ (applied gradually).

the plate occurs at between $Q=3.7$ and $Q=4$. By a refined estimation, we determined that $Q^{*}=3.75$ is the buckling critical value detected by the present method, which is very close to the critical value 3.7 given in [Okada et al. 1979].

When the lateral pressure is small $\left(Q \leq Q^{*}=3.75\right)$, the buckling does not occur; that is, there is neither the bifurcation point nor the unstable paths on the deflection-axial load curves. In fact, the deformation varies continuously towards the deflection form of triple bulges with the large value of $A_{3}$, with the increase of the edge compression from the single bulge deflection form.

When the lateral pressure is large $\left(Q>Q^{*}=3.75\right)$, the buckling occurs at a certain edge compression (the load at point A in Figure 10). In this case, the plate can show a jump behavior from a single bulge deformation form to a three bulges deformation form once the edge pressure reaches the critical value. In addition, an external stimulus to the plate may switch the deflection form of the plate when edge pressure is in between load of point A and load of point B, because in this interval (hysteresis area) this system has two stable states either of which is physically realizable.

Figure 11 provides the solutions for plates 4 and 5 by the present method and the Rayleigh-Ritz method [Okada et al. 1979]. It can be seen that both methods can detect the critical buckling points. However, there exists a discrepancy between the two methods. Similar to the case in Section 4.3, the differences between the solutions are due to the different sets of boundary conditions, although both of which might be loosely described as simply supported.

4.5. A rectangular plate subjected to shear and uniaxial compression. A rectangular plate under shear stress and uniaxial compression is analyzed with dimensions being $a=2, b=1$ and $t=0.009$. The uniaxial compression $P_{x}=0.65 P_{\text {xcr }}$ is exerted first and kept constant. The shear stress is applied incrementally from $0.1 \tau_{\text {cr }}$ to $2 \tau_{\text {cr }}$ with a step size $0.1 \tau_{\mathrm{cr}}$. The final deformation of the plate is shown in Figure 12. 


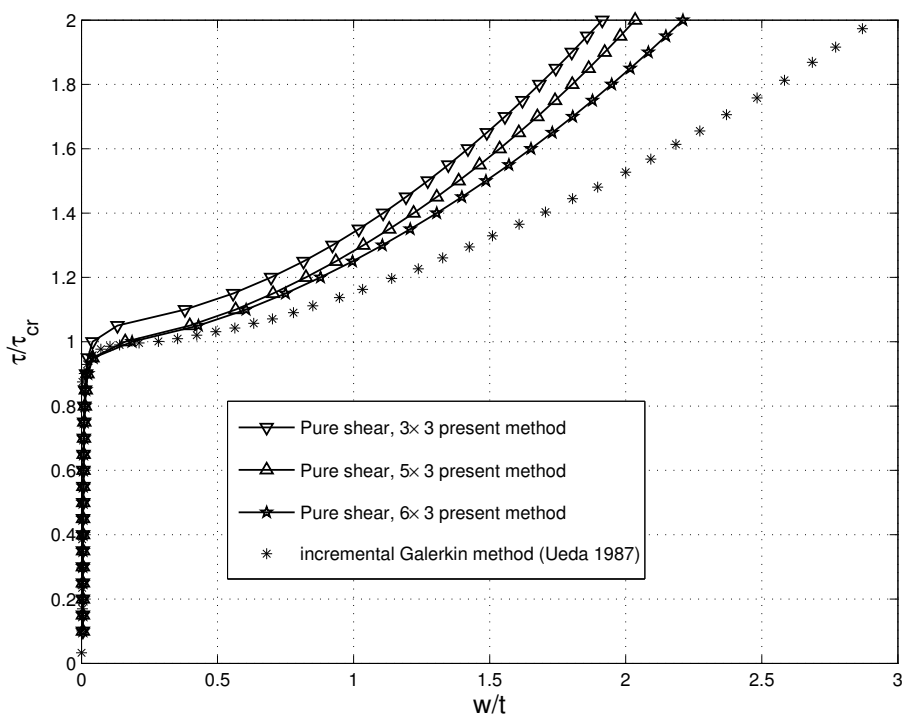

Figure 13. Comparisons of load-deflection curves as given by present global Galerkin method and the incremental Galerkin method [Ueda et al. 1987] under a pure shearing load.

Load-deflection curves of the rectangular plate under pure shearing load are plotted in Figure 13 via the present method and the incremental Galerkin method. It is shown that the present method is in accord with the incremental Galerkin method. The addition of terms in the assumed deflection function can generate a more accurate result if the result of the incremental Galerkin method is taken as a benchmark. Also, we can see that the deflection calculated by the present method is smaller than the incremental Galerkin method in general. It can be seen that the buckling critical value for the plate is about $\tau \approx \tau_{\mathrm{cr}}$, since there is no extra force acting on the plate and the initial imperfection is very slight so that it does not influence much.

Load-deflection curves of the plate under combined uniaxial and shearing loadings are given in Figure 14. A similar conclusion can be obtained as the above pure shearing case. There is a discrepancy between the results of the present method and the incremental Galerkin method. As the number of terms in the deflection function increases, the difference between the present method and the incremental method decreases. It shows from Figure 14 that the critical shearing value for the plate buckling decreases from the theoretical value $\tau_{\mathrm{cr}}$ of the pure shearing condition to approximately half of $\tau_{\mathrm{cr}}$ of the combined load condition. It illustrates that the uniaxial compression acting on the plate can degrade the critical value for shearing buckling. Plus, with rectangular plates, more terms in the longitudinal direction are required such that the real deflection shape of the plate can be satisfactorily described.

4.5.1. Comparison of solvers. The consumed computing efforts via different solvers are provided in Table 6, where eight term $(4 \times 2)$ deflection function is used in the present Galerkin method.

We see that the DNM2, that is, classical Newton method, is the fastest method both in terms of iteration numbers and computational time. The DNM1, a variant Newton method, is comparable with the Newton method. The DNMs are regarded as Newton-type methods, since they involve with the 


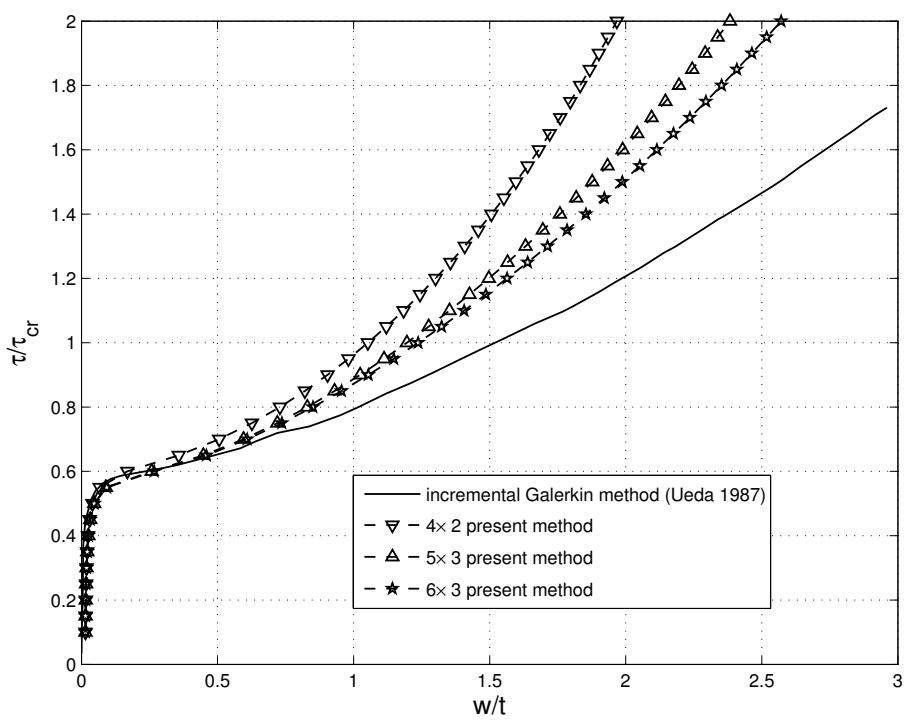

Figure 14. Comparisons of load-deflection curves as given by present global Galerkin method and the incremental Galerkin method [Ueda et al. 1987] under uniaxial compression combined with a shearing load.

\begin{tabular}{lcrc}
\hline method & time(s) & iterations & TPI \\
\hline MBECA1 & 174.07 & 17394 & 0.0100 \\
MBECA2 & 159.4 & 15938 & 0.0100 \\
DNM1 & 5.28 & 472 & 0.0112 \\
DNM2 & 5.05 & 457 & 0.0111 \\
DJIFM1 & 35.68 & 3479 & 0.0103 \\
DJIFM2 & 21.81 & 2143 & 0.0102 \\
\hline OVDA & 12.96 & 1298 & 0.0100 \\
OIA/ODV[R] & 95.03 & 9655 & 0.0098 \\
OIA/ODV[F] & 16.19 & 1689 & 0.0096 \\
OIA(R) & 12.93 & 1300 & 0.0099 \\
OIA(F) & 12.94 & 1300 & 0.0100 \\
GOIA & 12.94 & 1300 & 0.0100 \\
\hline
\end{tabular}

Table 6. Example 5: $4 \times 2$ present method.

inverse of Jacobian matrix. The TPIs of the DNM1 and DNM2 are larger than those of other methods, which can be explained by the need for inverting Jacobian matrix. In general, the iterative methods are several times faster than the continuous methods except the OIA/ODV[R]. However owing to the small size of the Jacobian matrix, the TPIs of DNMs are only slightly larger. Therefore the DNMs, which 
converge quadratically fast, are preferable choices for solving the current Galerkin-resultant system of NAEs which involve only a small number of nonlinear equations.

The prominent Jacobian-inverse-free methods, for example, OVDA, the OIAs and the GOIA, can be employed in solving nonlinear problems whose initial guess is hard to choose, whose Jacobian matrix is ill-conditioned or nearly singular, when the number of equations tends to be very large, and when the system of equations is either over or under determined.

\section{Conclusion}

In this paper a global Galerkin method is presented for solving the simply supported initially imperfect von Kármán plate under a combination of in-plane and lateral loads. The coupled nonlinear differential equations are transformed into a system of NAEs via the global Galerkin method. The explicit form of the Jacobian matrix ("tangent stiffness" matrix) of the NAEs is derived to eliminate the symbolic calculations involved in solving the NAEs. Plates subjected to uniaxial compression, or lateral pressure, or a combination of axial compression and lateral pressure, or a combination of shear stress and axial compression, are investigated by the present global Galerkin method in numerical examples. Large deflections of plate under different loading conditions are analyzed, and nonlinear phenomena such as the buckling behavior and the jump phenomenon are discussed. The present method is validated to be in agreement with the perturbation method, the incremental Galerkin method, the finite difference method, the finite element method and the experiments.

The present method is extremely advantageous provided that the deflection shape of a plate can be accurately expressed by the deflection function with a few terms. Numerical experiments indicate that the computing effort of the present method would be very economic when less than forty terms are considered in the assumed deflection function. Also, because of the extremely high accuracy provided at a very modest cost, the global Galerkin method may also provide the much needed highly accurate benchmark solutions against which other numerical methods may be validated. While distributed loads are treated in the present paper using the Galerkin method and trigonometric basis functions, (nonlinear) plates subjected to concentrated loads at arbitrary locations will be treated in a forthcoming paper using radial basis functions [Atluri 2005] and a spatial collocation or a Galerkin method. The resulting NAEs for these point load problems can be solved by the same methods as in the present paper.

On the other hand, a series of scalar homotopy methods (mainly the Newton homotopy methods), which do not need to invert the Jacobian matrix, are reviewed and used to solve the Galerkin-resulting system of NAEs. The performance of each method is evaluated. The GOIA, the OIAs and the OVDA promise to be the best Jacobian-inverse-free methods hitherto. A general form equation is proposed to incorporate all the existing scalar Newton homotopy methods in a uniform framework. Interestingly, the classical Newton method (labeled DNM2 in paper) can be generated from this general dynamical system under a certain condition.

\section{Acknowledgement}

This study is financially supported by the Doctorate Foundation of Northwestern Polytechnical University (CX201305), the Chinese NSF (11172235), and the Doctor Subject Foundation of the Ministry of Education of China (20106102110018). 


\section{References}

[Atluri 2005] S. N. Atluri, Methods of computer modeling in engineering and the sciences, I: A unified treatment of finite volume, finite element, field-boundary element, meshless, and boundary methods, Tech Science Press, Forsyth, GA, 2005.

[Atluri et al. 2009] S. N. Atluri, C.-S. Liu, and C.-L. Kuo, "A modified Newton method for solving non-linear algebraic equations", J. Marine Sci. and Tech. 17:3 (2009), 238-247.

[Bergan and Clough 1973] P. G. Bergan and R. W. Clough, "Large deflection analysis of plates and shallow shells using the finite element method", Int. J. Numer. Methods Engin. 5 (1973), 543-556.

[Bert et al. 1989] C. W. Bert, S. K. Jang, and A. G. Striz, "Nonlinear bending analysis of orthotropic rectangular plates by the method of differential quadrature", Comput. Mech. 5:2-3 (1989), 217-226.

[Brebbia and Connor 1969] C. Brebbia and J. Connor, "Geometrically nonlinear finite-element analysis", J. Engng. Mech. Div. (ASCE) 95 (1969), 463-483.

[Brown and Harvey 1969] J. C. Brown and J. M. Harvey, "Large deflections of rectangular plates subjected to uniform lateral pressure and compressive edge loading”, J. Mech. Engin. Sci. 11:3 (1969), 305-317.

[Chia 1980] C. Y. Chia, Nonlinear analysis of plates, McGraw-Hill, New York, NY, 1980.

[Dai et al. 2011a] H. H. Dai, J. K. Paik, and S. N. Atluri, "The global nonlinear Galerkin method for the analysis of elastic large deflections of plates under combined loads: a scalar homotopy method for the direct solution of nonlinear algebraic equations", Comput. Mater. Continua 23:1 (2011), 69-99.

[Dai et al. 2011b] H. H. Dai, J. K. Paik, and S. N. Atluri, "The global nonlinear Galerkin method for the solution of von Karman nonlinear plate equations: an optimal and faster iterative method for the direct solution of nonlinear algebraic equations $\boldsymbol{F}(\boldsymbol{x})=\mathbf{0}$, using $\boldsymbol{x}=\lambda\left[\alpha \boldsymbol{F}+(1-\alpha) \boldsymbol{B}^{T} \boldsymbol{F}\right]$ ", Comput. Mater. Continua 23:2 (2011), 155-185.

[Dai et al. 2014a] H. H. Dai, X. K. Yue, J. P. Yuan, and S. N. Atluri, "A time domain collocation method for studying the aeroelasticity of a two dimensional airfoil with a structural nonlinearity", J. Comput. Phys. 270 (2014), 214-237.

[Dai et al. 2014b] H. H. Dai, X. K. Yue, J. P. Yuan, and D. Xie, "A fast harmonic balance technique for periodic oscillations of an aeroelastic airfoil", 2014. Submitted for publication in J. Fluids Struct.

[Hirsch and Smale 1979] M. W. Hirsch and S. Smale, "On algorithms for solving $f(x)=0$ ", Comm. Pure Appl. Math. 32:3 (1979), 281-313.

[Kane and Levinson 1985] T. R. Kane and D. A. Levinson, Dynamics, theory and applications, McGraw-Hill, New York, NY, 1985.

[Ku and Yeih 2012] C.-Y. Ku and W. Yeih, "Dynamical Newton-like methods with adaptive stepsize for solving nonlinear algebraic equations", Comput. Mater. Continua 31:3 (2012), 173-200.

[Ku et al. 2009] C.-Y. Ku, W. Yeih, C.-S. Liu, and C.-C. Chi, "Applications of the fictitious time integration method using a new time-like function", CMES Comput. Model. Eng. Sci. 43:2 (2009), 173-190.

[Ku et al. 2011] C.-Y. Ku, W. Yeih, and C.-S. Liu, "Dynamical Newton-like methods for solving ill-conditioned systems of nonlinear equations with applications to boundary value problems", CMES Comput. Model. Eng. Sci. 76:2 (2011), 83-108.

[Levy 1942a] S. Levy, "Bending of rectangular plates with large deflections", technical report 737, National Advisory Committee for Aeronautics, 1942, http://naca.central.cranfield.ac.uk/reports/1942/naca-report-737.pdf.

[Levy 1942b] S. Levy, "Bending of rectangular plates with large deflections", technical note 846, National Advisory Committee for Aeronautics, 1942, http://www.dtic.mil/dtic/tr/fulltext/u2/a800848.pdf.

[Levy et al. 1944] S. Levy, D. Goldenberg, and G. Zibritosky, "Simply supported long rectangular plate under combined axial load and normal pressure", technical note 949, National Advisory Committee for Aeronautics, 1944, http://digital.library.unt.edu/ ark:/67531/metadc59844/m1/1/.

[Liu and Atluri 2008] C.-S. Liu and S. N. Atluri, "A novel time integration method for solving a large system of non-linear algebraic equations", CMES Comput. Model. Eng. Sci. 31:2 (2008), 71-83.

[Liu and Atluri 2011a] C.-S. Liu and S. N. Atluri, "An iterative algorithm for solving a system of nonlinear algebraic equations, $\boldsymbol{F}(\boldsymbol{x})=\mathbf{0}$, using the system of ODEs with an optimum $\alpha$ in $\dot{\boldsymbol{x}}=\lambda\left[\alpha \boldsymbol{F}+(1-\alpha) \boldsymbol{B}^{\boldsymbol{T}} \boldsymbol{F}\right] ; B_{i j}=\partial F_{i} / \partial x_{j}$ ", CMES Comput. Model. Eng. Sci. 73:4 (2011), 395-431. 
[Liu and Atluri 2011b] C.-S. Liu and S. N. Atluri, "Simple 'residual-norm' based algorithms, for the solution of a large system of non-linear algebraic equations, which converge faster than the Newton's method", CMES Comput. Model. Eng. Sci. 71:3 (2011), 279-304.

[Liu and Atluri 2012] C.-S. Liu and S. N. Atluri, "A globally optimal iterative algorithm using the best descent vector $\dot{\boldsymbol{x}}=$ $\lambda\left[\alpha_{c} \boldsymbol{F}+\boldsymbol{B}^{T} \boldsymbol{F}\right]$, with the critical value $\alpha_{c}$, for solving a system of nonlinear algebraic equations $\boldsymbol{F}(\boldsymbol{x})=\mathbf{0}$ ", CMES Comput. Model. Eng. Sci. 84:6 (2012), 575-602.

[Liu et al. 2009] C.-S. Liu, W. Yeih, C.-L. Kuo, and S. N. Atluri, "A scalar homotopy method for solving an over/underdetermined system of non-linear algebraic equations”, CMES Comput. Model. Eng. Sci. 53:1 (2009), 47-72.

[Liu et al. 2011a] C.-S. Liu, H.-H. Dai, and S. N. Atluri, "Iterative solution of a system of nonlinear algebraic equations $\boldsymbol{F}(\boldsymbol{x})=0$, using $\dot{\boldsymbol{x}}=\lambda[\alpha \boldsymbol{R}+\beta \boldsymbol{P}]$ or $\dot{\boldsymbol{x}}=\lambda\left[\alpha \boldsymbol{F}+\beta \boldsymbol{P}^{*}\right] \boldsymbol{R}$ is a normal to a hyper-surface function of $\boldsymbol{F}, \boldsymbol{P}$ normal to $\boldsymbol{R}$, and $\boldsymbol{P}^{*}$ normal to $\boldsymbol{F}$ ", CMES Comput. Model. Eng. Sci. 81:3-4 (2011), 335-363.

[Liu et al. 2011b] C.-S. Liu, H.-H. Dai, and S. N. Atluri, "A further study on using $\dot{\boldsymbol{x}}=\lambda[\alpha \boldsymbol{R}+\beta \boldsymbol{P}]\left(\boldsymbol{P}=\boldsymbol{F}-\boldsymbol{R}(\boldsymbol{F} \cdot \boldsymbol{R}) /\|\boldsymbol{R}\|^{2}\right)$ and $\dot{\boldsymbol{x}}=\lambda\left[\alpha \boldsymbol{F}+\beta \boldsymbol{P}^{*}\right]\left(\boldsymbol{P}^{*}=\boldsymbol{R}-\boldsymbol{F}(\boldsymbol{F} \cdot \boldsymbol{R}) /\|\boldsymbol{F}\|^{2}\right)$ in iteratively solving the nonlinear system of algebraic equations $\boldsymbol{F}(\boldsymbol{x})=0$ ", CMES Comput. Model. Eng. Sci. 81:2 (2011), 195-228.

[O'Donoghue and Atluri 1987] P. E. O'Donoghue and S. N. Atluri, "Field/boundary element approach to the large deflection of thin flat plates", Comput. Struct. 27:3 (1987), 427-435.

[Okada et al. 1979] H. Okada, K. Oshima, and Y. Fukumoto, "Compressive strength of long rectangular plates under hydrostatic pressure", J. Soc. Naval Arch. Japan 146:1 (1979), 270-280.

[Paik et al. 2001] J. K. Paik, A. K. Thayamballi, and B. Ju Kim, "Large deflection orthotropic plate approach to develop ultimate strength formulations for stiffened panels under combined biaxial compression/tension and lateral pressure", ThinWalled Struct. 39:3 (2001), 215-246.

[Punch and Atluri 1986] E. F. Punch and S. N. Atluri, "Large displacement analysis of plates by a stress-based finite element approach”, Comput. Struct. 24:1 (1986), 107-117.

[Ramm 2004] A. G. Ramm, "Dynamical systems method for solving operator equations", Commun. Nonlinear Sci. Numer. Simul. 9:4 (2004), 383-402.

[Shen 1989] H. S. Shen, "Postbuckling behaviour of rectangular plates under combined loading", Thin-walled Struct. 8:3 (1989), 203-216.

[Timoshenko 1961] S. P. Timoshenko, Theory of elastic stability, 2nd ed., McGraw-Hill Book Co., New York, 1961.

[Ueda et al. 1987] Y. Ueda, M. H. Rashed, and J. K. Paik, "An incremental Galerkin method for plates and stiffened plates", Comput. Struct. 27:1 (1987), 147-156.

[Von Kármán 1910] T. Von Kármán, "Festigkeitsprobleme im maschinenbau”, Encykl. Math. Wiss. 4 (1910), 314-385.

[Way 1939] S. Way, "Uniformly loaded, clamped, rectangular plates with large deflection", pp. 123-128 in Proceedings of the Fifth International Congress of Applied Mechanics (Cambridge, MA), Wiley, Cambridge, MA, 1939.

[Woolley et al. 1946] R. M. Woolley, J. N. Correick, and S. Levy, "Clamped long rectangular plate under combined axial load and normal pressure", technical note 1047, National Advisory Committee for Aeronautics, 1946, http://www.dtic.mil/dtic/tr/ fulltext/u2/a800975.pdf.

[Yamamoto et al. 1970] Y. Yamamoto, N. Matsubara, and T. Murakami, "Buckling of plates subjected to edge thrusts and lateral pressure", J. Soc. Naval Arch. Japan 127 (1970), 171-179. In Japanese.

Received 16 Oct 2013. Revised 8 Mar 2014. Accepted 7 Apr 2014.

HonghuA DAI: daihonghua@gmail.com

College of Astronautics, Northwestern Polytechnical University, Xi'an 710072, China

XIAOKUi YUE: xkyue@nwpu.edu.cn

College of Astronautics, Northwestern Polytechnical University, Xi' an 710072, China

SATYA N. ATLURI: satluri@uci.edu

Center for Aerospace Research and Education, University of California, Irvine, Irvine, CA 92697, United States 


\title{
JOURNAL OF MECHANICS OF MATERIALS AND STRUCTURES
}

\author{
msp.org/jomms
}

\author{
Founded by Charles R. Steele and Marie-Louise Steele
}

EDITORIAL BOARD

ADAIR R. AGUIAR

KATIA BERTOLDI

University of São Paulo at São Carlos, Brazil

DAVIDE BIGONI

Harvard University, USA

IWONA JASIUK

University of Trento, Italy

Thomas J. PENCE

University of Illinois at Urbana-Champaign, USA

YASUHIDE SHINDO

Michigan State University, USA

DAVID STEIGMANN

Tohoku University, Japan

University of California at Berkeley

\section{ADVISORY BOARD}

J. P. CARTER University of Sydney, Australia

R. M. Christensen Stanford University, USA

G. M. L. GLADWELL University of Waterloo, Canada

D. H. Hodges Georgia Institute of Technology, USA

J. HUTCHINSON Harvard University, USA

C. HwU National Cheng Kung University, Taiwan

B. L. KARIHALOO University of Wales, UK

Y. Y. KIM Seoul National University, Republic of Korea

Z. Mroz Academy of Science, Poland

D. PAMPlonA Universidade Católica do Rio de Janeiro, Brazil

M. B. RubiN Technion, Haifa, Israel

A. N. SHUPIKOV Ukrainian Academy of Sciences, Ukraine

T. TARNAI University Budapest, Hungary

F. Y. M. WAN University of California, Irvine, USA

P. WRIGGERS Universität Hannover, Germany

W. YANG Tsinghua University, China

F. ZIEGLER Technische Universität Wien, Austria

PRODUCTION production@msp.org

SILVIO LEVY Scientific Editor

Cover photo: Mando Gomez, www.mandolux.com

See msp.org/jomms for submission guidelines.

JoMMS (ISSN 1559-3959) at Mathematical Sciences Publishers, 798 Evans Hall \#6840, c/o University of California, Berkeley, CA 94720-3840, is published in 10 issues a year. The subscription price for 2014 is US \$555/year for the electronic version, and $\$ 710 /$ year (+\$60, if shipping outside the US) for print and electronic. Subscriptions, requests for back issues, and changes of address should be sent to MSP.

JoMMS peer-review and production is managed by EditFLow ${ }^{\circledR}$ from Mathematical Sciences Publishers.

PUBLISHED BY

mathematical sciences publishers

nonprofit scientific publishing

http://msp.org/

(C) 2014 Mathematical Sciences Publishers 


\title{
Journal of Mechanics of Materials and Structures
}

\author{
Volume 9, No. $2 \quad$ March 2014
}

Plane waves at the boundary of two micropolar thermoelastic solids with distinct conductive and thermodynamic temperatures

Rajneesh Kumar, Mandeep Kaur and Satish C. RaJvanshi

Dynamic compression of square tube cellular structures RYAN L. HOLLOMAN, KaRTHIKEyan Kandan, VikRam DeshPande and HaYdn N. G. WadLey

Dynamic response of twin lined shells due to incident seismic waves

J. P. DWIVEdi, V. P. Singh and RADHA KRISHNA LAL 183

Solutions of the von Kármán plate equations by a Galerkin method, without inverting the tangent stiffiness matrix.

Honghua Dai, Xiaokuí Yue and Satya N.Atluri 195

Bimaterial lattices with anisotropic thermal expansion

Marina M. Toropova and Craig A. Steeves

Origin and effect of nonlocality in a composite STEWART A. Silling 